\title{
The Science and Experimental Equipment for
}

\section{The $12 \mathrm{GeV}$ Upgrade of CEBAF}

\section{Updated from the Document Originally Prepared for the April 2005 DOE Science Review}

\author{
Jefferson Lab \\ January 10, 2005
}

\begin{abstract}
Jefferson Lab is managed and operated by Jefferson Science Associates, LLC (JSA) for the Office of Science of the U.S. Department of Energy.
\end{abstract}

Thomas Jefferson National Accelerator Facility 12000 Jefferson Avenue

Newport News, VA 23606

www.JLab.org

(757)-269-7100 



\section{Introduction}

This document presents a summary of the scientific motivations for the $12 \mathrm{GeV}$ Upgrade of the CEBAF facility at Jefferson Lab. It is based on the document originally prepared for the April 2005 DOE Science Review of the project, and incorporates relatively minor edits and updates. We will revise it more throughly following the discussions expected to take place at the APS Division of Nuclear Physics Town Meeting on QCD and Hadron Physics to be held in Rutgers, NJ January 12-14, 2007 as part of the NSAC 2007 Long Range Plan process.

The 12 GeV CEBAF Upgrade of Jefferson Lab received Critical Decision Zero (CD-0) approval in March 2004. This affirmed that the Department of Energy had approved the mission need of the project which allowed Jefferson Lab scientists to proceed with the conceptual design and to request funding. The upgraded facility will double the maximum electron beam energy to $12 \mathrm{GeV}$, which will be delivered to a new experimental hall, and it will also deliver up to an $11 \mathrm{GeV}$ beam to the three existing halls, each with increased experimental capabilities.

In support of the review process for the next critical decision, Jefferson Lab and the user community further defined and updated the scientific motivation. The Office of Nuclear Physics conducted a Science Review of the $12 \mathrm{GeV}$ Upgrade in April 2005 using a panel of international experts to assess the major elements of the science program: gluonic excitations, the fundamental structure of hadrons, the physics of nuclei, and fundamental symmetry tests in nuclear physics. The panel concluded that the research is "unique to TJNAF and will not be possible at any other known facility in the foreseeable future", and that "the scientific opportunity afforded by the $12 \mathrm{GeV}$ Upgrade is outstanding, providing the U.S. with unique world-leadership capabilities in studies of QCD and the quark structure of matter." Both the search for exotic mesons and the study of fundamental symmetries were noted as having "discovery potential."

The project received CD-1 (Approve Alternative Selection and Cost Range) in February 2006 with an announcement made on Feb. 22nd by the Secretary of Energy, Samuel W. Bodman, during a visit to Jefferson Lab. The Project Engineering and Design (PED) phase is underway. DOE has just completed a progress review of the project (held January 9-10, 2007) and concluded that there has been significant progress and the "12 GeV Upgrade Project is on track in their preparations and readiness for CD-2 approval in September 200\%." CD-3 (Approve Construction Start) is expected in late 2008. The Upgrade is Jefferson Lab's highest priority; it enables a cutting-edge science program for the laboratory's broad-based user community for the next generation of experiments.

The Jefferson Lab Program Advisory Committee (PAC) has played a critical role in the evolution of the science case for the new facility and the planning for initial complement of experimental equipment. PACs 18, 23 and 27 each reviewed the developing science case for the Upgrade and the equipment proposed to realize it, and this document reflects both the hard work of the JLab community and substantial peer review and recommendations from the PAC. Since the science review took place, we held the first meeting of the PAC that formally considered proposed experiments for the Upgrade (PAC30); it took place August 21-26, 2006. A total of 22 proposals and 8 letters of intent for experiments that will use the base equipment currently planned for the Upgrade were submitted by JLab users, and the PAC recommended approval (or conditional approval) of seventeen of them. They are listed in the Appendix to this document, and the detailed proposals are available on the JLab website at http://www.jlab.org/exp_prog/proposals/06prop.html. 
To quote from the PAC report:

The PAC heard excellent talks covering a physics program that reflected almost a decade of discussions and preparations focused on the physics possibilities opened by the availability of 11-12 GeV beam at JLab. The basis for those discussions has been the tremendous progress at the laboratory and in the community in both experiment and theory. On the experimental side the implementation and reliable operation of highly polarized, intense beams and polarized proton and neutron targets, complemented with efficient proton recoil polarization capabilities opened up new directions for investigations. Together with very specialized, sophisticated detector systems a broad range of physics questions could be addressed. The precision of the observables extracted from the data inspired new developments on the theory side. The dynamics of extensive physics discussions during the years of preparation became transparent for the PAC members during the talks and, especially, during extended discussions with the proponents of the proposals. We noted with particular pleasure the large number of young spokespersons who gave excellent presentations.

With this review, the decision process necessary to finalize and prioritize the initial science program for the $12 \mathrm{GeV}$ Upgrade is formally underway. 


\section{Abstract}

This Conceptual Design Report (CDR) presents the compelling scientific case for upgrading the Continuous Electron Beam Accelerator Facility (CEBAF) at Jefferson Lab to $12 \mathrm{GeV}$. Such a facility will make profound contributions to the study of hadronic matter. In particular, it will allow breakthrough programs to be launched in four main areas:

- The experimental study of gluonic excitations in order to understand the fundamentally new dynamics that underpins all of nuclear physics: the confinement of quarks. Theoretical conjectures, now strengthened by lattice QCD simulations, indicate that the most spectacular new prediction of QCD - quark confinement - occurs through the formation of a string-like "flux tube" between quarks. This conclusion (and proposed mechanisms of flux tube formation) can be tested by determining the spectrum of the gluonic excitations of mesons.

- The Fundamental Structure of the Nuclear Building Blocks. A vast improvement in our knowledge of the fundamental structure of the proton and neutron can be achieved. Not only can existing "deep inelastic scattering" cross sections be extended for the first time to cover the critical region where their basic three-quark structure dominates, but also measurements of new "deep exclusive scattering" cross sections will open the door to a comprehensive characterization of these wavefunctions using the framework of the Generalized Parton Distributions; these data will provide access to information on the correlations among the quarks. These studies will be complemented by detailed measurements of elastic and transition form factors, determining the dynamics underlying the quark-gluon structure through measurements of their high-momentum-transfer behavior and providing essential constraints on their description.

- The Physics of Nuclei. A broad and diversified program of measurements that (taken together with the hadron studies outlined above) aims to provide a firm intellectual underpinning for all of nuclear physics by answering the question "How does the phenomenological description of nuclei as nucleons interacting via an effective interaction parameterized using meson exchange arise from the underlying dynamics of quarks and gluons?" It has two main components:

The emergence of nuclei from QCD. Experiments aimed at understanding how the description of nuclei in terms of nucleons interacting via the $N-N$ force arises from the more fundamental QCD description. It includes the investigation of the partonic structure of nuclei, of short range structures in nuclei, and of the modification of the quark-gluon structure of the nucleons and mesons by the nuclear environment.

Fundamental QCD processes in the nuclear arena. Experiments aimed at understanding how hadron-hadron interactions arise from the underlying quark-gluon structure of QCD.

- Tests of the Standard Model of electro-weak interactions and the determination of fundamental parameters of this model. Precision, parity-violating electron scattering experiments made feasible by the $12 \mathrm{GeV}$ Upgrade have the sensitivity to search for deviations from the Standard Model that could signal the presence of new physics. Precision measurements of the two-photon decay widths and transition form factors of the three neutral pseudoscalar mesons $\pi^{0}, \eta$, and $\eta^{\prime}$ via the Primakoff effect will lead to a significant improvement on our knowledge of chiral symmetry in QCD, in particular on the ratios of quark masses and on chiral anomalies.

This science program has expanded significantly since the project was first presented to the Nuclear Sciences Advisory Committee (NSAC) in the form of a White Paper [WP01] produced as part of the 2001-2002 NSAC Long Range Planning Process and since the pre-Conceptual Design Report (pCDR) produced in June 2004 to document the Upgrade science and experimental equipment plans [pCDR]. While focusing on science, this document also provides a brief description of the required detector and accelerator upgrades so that it can serve as an overview of the entire plan for the $12 \mathrm{GeV}$ project. 
This CDR was developed from the documentation presented in the pCDR [pCDR] and further discussions within the community since that document was released. We acknowledge here the many contributions of the entire JLab community, and note that the author list at the end of the pCDR includes the names of all contributors to the effort known to us. Many of them commented extensively on the earlier drafts, resulting in a much-improved document. This document would have been impossible without their intelligence, enthusiasm, time, and just plain hard work.

\section{The 12 GeV Upgrade CDR Editorial Board:}

John Arrington, Argonne National Lab (johna@anl.gov)

Ted Barnes, Oak Ridge National Lab (barnesfe@ornl.gov) Aron Bernstein, Massachusetts Institute of Technology (bernstein@mitlns.mit.edu) Will Brooks, Jefferson Lab (brooksw@jlab.org)

Volker Burkert, Jefferson Lab (burkert@jlab.org)

Lawrence Cardman, Jefferson Lab (cardman@jlab.org) Carl Carlson, College of William \& Mary (carlson@physics.wm.edu) Gordon Cates, University of Virginia (cates@Virginia.EDU)

J.-P. Chen, Jefferson Lab (jpchen@jlab.org)

Alex Dzierba, Indiana University (dzierba@indiana.edu) Rolf Ent, Jefferson Lab (ent@jlab.org)

Latifa Elouadrhiri, Jefferson Lab (latifa@jlab.org) Howard Fenker, Jefferson Lab (hcf@jlab.org)

Haiyan Gao, Duke University (gao@tunl.duke.edu) Ashot Gasparian, North Carolina A \& T/Jefferson Lab (gasparan@jlab.org) Jose Goity, Hampton University/Jefferson Lab (goity@jlb.org)

Doug Higinbotham, Jefferson Lab (doug@jlab.org)

Roy Holt, Argonne National Lab (holt@anl.gov)

Charles Hyde-Wright, Old Dominion University (chyde@odu.edu)

Kees de Jager, Jefferson Lab (kees@jlab.org)

Sabine Jeschonnek, Ohio State University (jeschonnek.L@osu.edu)

Xiangdong Ji, University of Maryland (xji@physics.umd.edu)

Xiaodong Jiang, Rutgers University (jiang@jlab.org)

Mark Jones, Jefferson Lab (jones@jlab.org)

Thia Keppel, Hampton University/Jefferson Lab (keppel@jlab.org)

Sebastian Kuhn, Old Dominion University (skuhn@odu.edu)

Krishna Kumar, University of Massachusetts (kkumar@physics.umass.edu)

Jean-Marc Laget, Centre d'Etudes Nucleaire, Saclay, and Jefferson Lab (laget@jlab.org)

Dave Mack, Jefferson Lab (mack@jlab.org)

Curtis Meyer, Carnegie Mellon University (cmeyer@ernest.phys.cmu.edu)

Wally Melnitchouk, Jefferson Lab (wmelnitc@jlab.org)

Zein-Eddine Meziani, Temple University (meziani@temple.edu)

Anatoly Radyushkin, Old Dominion University/Jefferson Lab (radyush@jlab.org) Mike Ramsey-Musolf, California Institute of Technology (mjrm@krl.caltech.edu)

Paul Reimer, Argonne National Lab (reimer@anl.gov)

David Richards, Jefferson Lab (dgr@jlab.org)

Oscar Rondon, University of Virginia (rondon@Virginia.edu)

Carlos Salgado, Norfolk State University/Jefferson Lab (salgado@jlab.org)

Elton Smith, Jefferson Lab (elton@jlab.org)

Rocco Schiavilla, Old Dominion University/Jefferson Lab (schiavil@jlab.org)

Paul Souder, Syracuse University (souder@suhep.phy.syr.edu)

Paul Stoler, Rensselear Polytechnic Institute (stoler@rpi.edu)

Anthony Thomas, Jefferson Lab (awthomas@jlab.org)

Paul Ulmer, Old Dominion University (pulmer@odu.edu)

Larry Weinstein, Old Dominion University (lweinste@odu.edu)

Christian Weiss, Jefferson Lab (weiss@jlab.org)

Bogdan Wojtsekhowski, Jefferson Lab (bogdanw@jlab.org) 


\section{Contents}

1 Physics Overview 1

1.A Mapping Gluonic Excitations and Understanding Confinement . . . . . . . . . 2

Flux tubes and confinement. . . . . . . . . . . . . . . . 2

Conventional $q \bar{q}$ mesons. . . . . . . . . . . . . . . . . . . . . 3

Glueballs and hybrid mesons. . . . . . . . . . . . . . . . . 3

Photon probes and exotic hybrid mesons. . . . . . . . . . . . . . . . . 4

Identifying the spectrum of exotic hybrid mesons. . . . . . . . . . . . . 5

Spectroscopy of conventional mesons including strangeonium. . . . . . . . . . 5

Summary. . . . . . . . . . . . . . . . . . . . 7

1.B The Fundamental Structure of the Nuclear Building Blocks . . . . . . . . . . . 7

3D quark/gluon imaging of the nucleon. . . . . . . . . . . . . . . . . 9

Nucleon elastic form factors. . . . . . . . . . . . . . . . . 11

GPD's in deeply-virtual Compton scattering. . . . . . . . . . . . . . 12

GPD's in deeply-virtual meson production and other processes. . . . . 15

Resonance transition form factors and GPD's. . . . . . . . . . . . 16

The charged pion form factor. . . . . . . . . . . . . . 16

Valence quark structure. . . . . . . . . . . . . . . . . . . 17

Valence quark spin distributions at large $x \ldots \ldots \ldots 17$

Flavor structure of valence quarks at large $x \ldots \ldots 18$

Spin structure of the nucleon. . . . . . . . . . . . . . . . . . 18

Flavor decomposition of quark spin. . . . . . . . . . . . . . 20

Quark transverse spin distributions and quark transverse momentum in semi-inclusive scattering. . . . . . . . . . . . . . 20

The parton-hadron transition. . . . . . . . . . . . . . . 21

Quark-hadron duality. . . . . . . . . . . . . . . 22

Structure function moments and the Gerasimov-Drell-Hearn integral. 22

1.C The Physics of Nuclei . . . . . . . . . . . . . . . . . . . 23

The Emergence of Nuclei from QCD. . . . . . . . . . . . . . . . . . . 24

The partonic structure of nuclei. . . . . . . . . . . . . . . 24

Short-range structure of nuclei. . . . . . . . . . . . . . 26 
The onset of scaling behavior in nuclear reactions. . . . . . . . . . 26

Fundamental QCD processes in the nuclear arena. . . . . . . . . . . . . . . 29

Quark propagation through cold QCD matter: nuclear hadronization and transverse momentum broadening. . . . . . . . . . 29

Color transparency. . . . . . . . . . . . . . . . . . . . 31

1.D Symmetry Tests in Nuclear Physics . . . . . . . . . . . . . . . . . . . 31

Standard model tests. . . . . . . . . . . . . . . . . . . 31

Tests of Chiral symmetry and anomalies via the Primakoff effect. . . . . . . . 33

2 Upgrade Project Summary $\quad 35$

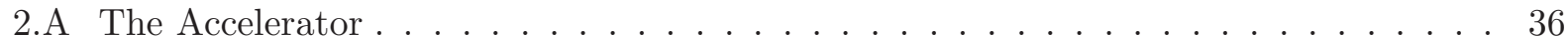

2.B The Experimental Equipment . . . . . . . . . . . . . . . . 38

Hall A and the High Resolution Spectrometers (HRS) . . . . . . . . . . . 38

Hall B Upgrade and CLAS12. . . . . . . . . . . . . . . . . . . . . . 39

Forward detector: . . . . . . . . . . . . . . . . 40

Central detector: . . . . . . . . . . . . . . . . . . 40

Beamline and targets: . . . . . . . . . . . . . . 4 40

Hall C and the Super High Momentum Spectrometer (SHMS). . . . . . . . . 41

Hall D and the GlueX Experiment. . . . . . . . . . . . . . . . . . . . 43

APPENDIX - PAC30 Recommended Proposals for 12 GeV Science 45

$\begin{array}{ll}\text { REFERENCES } & 46\end{array}$ 


\section{Physics Overview}

This Conceptual Design Report provides a summary of the science motivating the $12 \mathrm{GeV}$ Upgrade of CEBAF and the associated experimental equipment. Further details are available in the pre-Conceptual Design Report [pCDR]. The research program of the new facility is focused on four major research themes that coincide with broad directions of the field of nuclear physics as identified in two key documents: the 2002 Long Range Plan [NS02] of the Nuclear Science Advisory Committee of the U.S. Department of Energy and the National Science Foundation, and the recent decadal survey [NA99] of the field by the National Research Council of the National Academy of Sciences. We identify these themes here to place our research program in this broader context. Each addresses outstanding questions in nuclear physics that the laboratory's users address with a concerted program of experimental and theoretical work.

\section{Mapping Gluonic Excitations and Understanding Confinement}

Experiments and theory aimed at examining the fundamentally new dynamics that underpins all of nuclear physics: the confinement of quarks.

\section{How are the Nuclear Building Blocks Made from Quarks and Gluons?}

A program of measurements addressing the first question that must be answered in the quest to understand nuclear physics in terms of the fundamental theory of strongly interacting matter: quantum chromodynamics (QCD).

\section{The Physics of Nuclei}

Two broad programs that aim at understanding how nucleon-based models of nuclei with interacting nucleons and mesons arise as an approximation to the underlying quarkgluon structure of these systems, and understanding the QCD basis for the $N-N$ force. In addressing these fundamental questions, these programs will use the precision, spatial resolution, and interpretability of electromagnetic interactions to probe nuclear systems to distance scales where their underlying quark-gluon structure becomes evident.

\section{In Search of the New Standard Model}

Experiments searching for physics beyond the Standard Model of electro-weak interactions through precision tests of its predictions, and by measuring low energy parameters of the theory to deepen our understanding of chiral symmetry breaking.

Each of these programs is a major motivation for the energy upgrade. The first, a program of gluonic spectroscopy, will provide data needed: i) to establish the existence and mass spectrum of exotic mesons, and thereby: ii) to test experimentally our current understanding that quark confinement arises from the formation of QCD flux tubes. If our present understanding is incorrect, the experiment has the sensitivity necessary to decisively test first-principles lattice QCD calculations of the mesons - the simplest of the strongly-interacting systems. The second program will explore the complete structure of the nucleons through measurements: i) of quark momentum distributions in the critical, but poorly known, valence quark region; and ii) of exclusive reactions that build on the framework of the newly discovered Generalized Parton Distributions. The third will address outstanding issues in nuclear physics, completing a very fruitful area presently under investigation with $\mathrm{CEBAF}$ at $6 \mathrm{GeV}$ and extending this program in important new directions. Finally, the last program will use precision measurements to explore the validity of the Standard Model of electroweak interactions and measure key parameters of that theory. In Sections 1.A through 1.D we summarize these four key science drivers of the $12 \mathrm{GeV}$ Upgrade. Section 2 then completes the picture by summarizing the accelerator and experimental equipment upgrades required to accomplish these physics goals. 


\section{A Mapping Gluonic Excitations and Understanding Confinement}

The failure to observe isolated quarks or gluons, despite numerous searches, provides overwhelming experimental evidence that quarks and gluons are confined in nature. An understanding of how this confinement comes about in QCD is an outstanding fundamental question in physics. The confinement mechanism operates when the separation between quarks is large, $\gtrsim 1 \mathrm{fm}$. When the separation is small, the quarks behave as if they are free, and the understanding of this asymptotic freedom regime of QCD was recognized with the award of the 2004 Nobel Prize in Physics. Although quarks and gluons do not exist in isolation, their bound states, hadrons, are observed, and the prediction along with the experimental validation of their spectrum should provide a direct test of our understanding of confinement. But the most direct tests will come from a mapping of those hadrons that exhibit explicit gluonic degrees-of-freedom since the gluons mediate the force between quarks. QCD predictions about the spectrum of such hadrons is tied to how well the mechanism of confinement is understood so the experimental information on this spectrum is essential. The goal of the GlueX experiment is to map these states starting with exotic hybrid mesons that possess a unique signature distinguishing them from ordinary hadrons. Exotic hybrid mesons are expected to be more readily produced in photon, as opposed to pion or kaon induced interactions. High quality data on meson production with photons is sparse compared to extant data on hadroproduction of mesons. The energy upgrade of the CEBAF accelerator to $12 \mathrm{GeV}$ will provide a $9 \mathrm{GeV}$ linearly polarized photon beam into the GlueX detector in a new experimental hall (Hall D) optimized to identify properties of mesons produced in the mass range where exotic mesons are expected to occur. This combination of photon beam and detector will yield unprecedented statistics and precision heretofore unavailable, and will provide a unique and important discovery potential for GlueX.

Flux tubes and confinement. There is growing evidence from first-principles calculations of QCD on the lattice (LQCD) that as the quark separation in a hadron grows the energy required to separate the quarks increases linearly with distance. In a meson, for example, the quark $(q)$ and anti-quark $(\bar{q})$ are sources or sinks of color electric flux in analogy with ordinary electric charges and electric flux. Unlike ordinary electric flux, the color electric flux is expelled from the vacuum and trapped in a flux tube connecting the $q$ and $\bar{q}$ (see Fig. 1(a)).

It has been pointed out that this is similar to the way a superconductor expels ordinary magnetic flux and traps it in thin tubes called Abrikosov-Gorkov vortex lines. The formation of these color electric flux tubes is related to the ability of gluons to self-interact through their color charge. In contrast, for ordinary electric flux the field quanta, photons, do not self-interact and thus do not form flux tubes. Figure 1(b) shows a LQCD calculation of the action density in the color field in the vicinity of a $q$ and $\bar{q}$. The energy density peaks at the positions of the quarks and is confined inside a flux tube in between. Figure 1(c) shows the corresponding potential between the $q$ and $\bar{q}$ as a function of their separation $r$. At large values of $r$ the potential is linear in $r$ while for smaller $r$ the potential is Coulombic. This LQCD calculation is for heavy quarks in the quenched approximation. More recent unquenched calculations show similar results. There is also an analogy between the notion of a flux tube and notion of a relativistic string connecting the quarks in a hadron. Indeed, Y. Nambu first introduced this idea to explain the so-called linear Regge trajectories for light hadrons whereby the square of the hadron masses and their spins are linearly related, i.e. $m^{2} \propto J$. If one assumes the mass of the hadron is carried in the relativistic rotating string with massless quarks at its ends, the linear relation between $J$ and $m^{2}$ follows, 

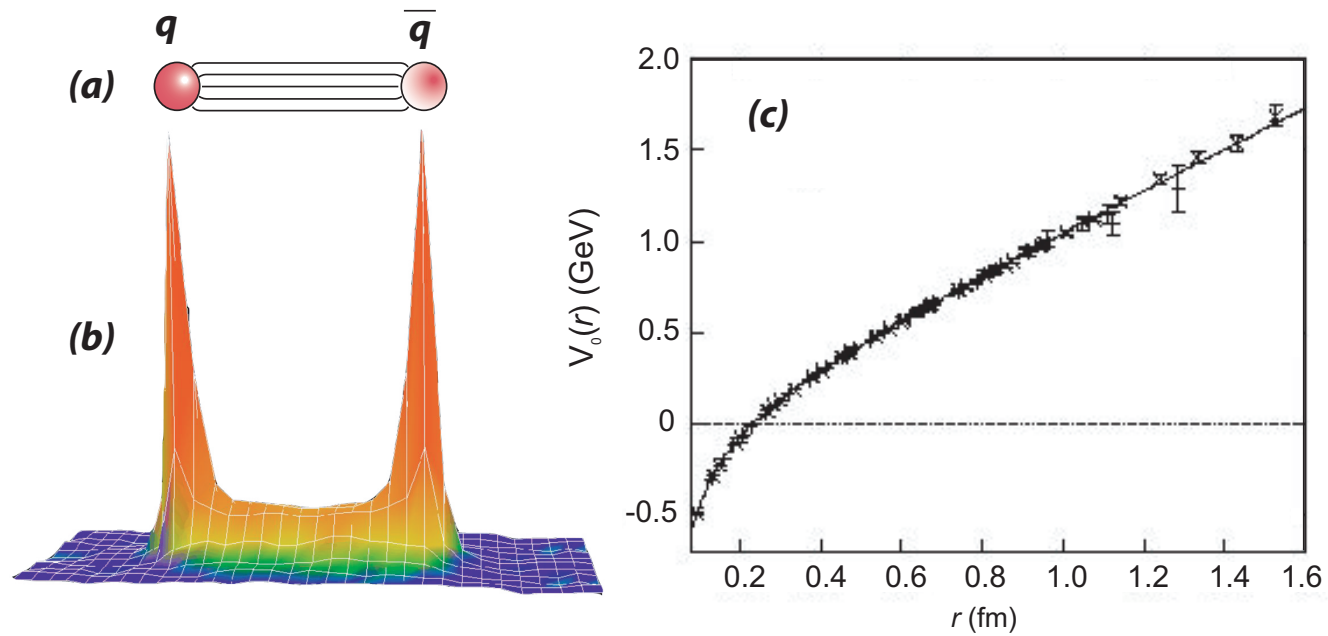

Figure 1: (a) The flux of chromo-electric field lines between a quark $(q)$ and anti-quark $(\bar{q})$ is confined to a flux-tube; (b) LQCD prediction (from G. Bali) of the action density in the color field in the space surrounding a $q$ and $\bar{q}$ showing the energy density peaking at the position of the quarks and confined to a flux tube between the quarks; (c) The corresponding potential (also from Bali) between the $q$ and $\bar{q}$ as a function of separation $r$. For large $r$ the potential is linear while for small $r$ it is Coulombic. The LQCD calculation by Bali is for heavy quarks in the quenched approximation. Recent unquenched calculations reach the same conclusions.

but only if the energy per unit length carried in the string is constant, which is equivalent to the assumption of a linearly rising potential.

Conventional $q \bar{q}$ mesons. In the conventional quark model of hadrons, there is no need to invoke gluonic degrees of freedom to explain the observed spectrum of low-lying mesons composed of up $(u)$, down $(d)$ and strange $(s)$ quarks - the flux tube is in its ground state. For a given total spin $\left(\vec{S}=\vec{S}_{q}+\vec{S}_{\bar{q}}\right)$ and relative orbital angular momentum $\left(\vec{L}=\vec{L}_{q \bar{q}}\right)$ we expect a nonet of mesons, all with the same $J^{P C}$ quantum numbers specified by the rules for a fermion-antifermion system. These rules give $\vec{J}=\vec{L}+\vec{S}, P=(-1)^{L+1}$ and $C=(-1)^{L+S} . J^{P C}$ combinations such as $0^{--}, 0^{+-}, 1^{-+}, 2^{+-}, \ldots$ are not allowed and are called exotic. Within a nonet one expects to find flavor states characterized by combining the flavors of $u, d$ and $s$ quarks into $q \bar{q}$ combinations to yield an iso-triplet with zero strangeness, two iso-doublets with strangeness +1 and -1 , and two strangeness zero iso-singlets. Consistent with this model, none of the known mesons carry flavor exotic quantum numbers (e.g. doubly charged or strangeness +1 with negative charge). Many positions in the low-lying nonets are assigned to known mesons. For the scalar mesons, there is some evidence that there are more mesons than can be accommodated within the quark model. In addition, there is some weak evidence that exotic $J^{P C}$ mesons might have been discovered. That mesons beyond simple $q \bar{q}$ bound states might exist is not inconsistent with QCD - indeed a richer spectrum of mesons is expected.

Glueballs and hybrid mesons. One such family of QCD mesons consisting solely of selfinteracting gluons with no quark content are called glueballs, and there may be some experimental evidence of such states. Since the quantum numbers of low-lying glueballs are not expected to be 
exotic, they should manifest themselves as extraneous states that cannot be accommodated within $\bar{q} q$ nonets. But their identification is complicated by the fact that they can mix with $\bar{q} q$. The current CLEO-c program at Cornell will culminate in the collection of data from 1 billion decays of the $J / \psi$ meson, whose radiative decays are expected to be rich in glueball production.

Another family of QCD mesons are those in which the flux tube binding the $q$ and $\bar{q}$ in a meson is excited. These are called hybrid mesons. Glueballs and hybrid mesons are collectively referred to as gluonic excitations. Hybrid mesons are expected to populate nonets characterized by a given $J^{P C}$ that is determined by the quantum numbers of both the excited flux tube and the quarks rather than just the quantum numbers of the quarks. For hybrid mesons exotic $J^{P C}$ are possible, providing a unique signature for the explicit role of glue. The spectroscopy of these exotic hybrid states is not complicated by possible mixing with conventional $q \bar{q}$ mesons. The level splitting between the ground state flux tube (conventional $q \bar{q}$ mesons) and the first excited transverse modes is $\pi / r$, where $r$ is the separation between the quarks. Based on this the hybrid spectrum should lie about $1 \mathrm{GeV} / c^{2}$ above the ground state spectrum.

According to the flux tube model and LQCD calculations, one expects the lightest $J^{P C}=1^{-+}$ exotic hybrid to have a mass of order $1.9 \mathrm{GeV} / c^{2}$ with the $J^{P C}=2^{+-}$exotic hybrid lying 0.2 to $0.5 \mathrm{GeV} / c^{2}$ higher (the uncertainties are large) in mass. A common feature of models describing hybrid decays is the selection rule (though not absolute) that the hybrid meson cannot transfer its angular momentum to the final state meson pairs as relative angular momentum, but instead to the internal angular momentum of the $q \bar{q}$ pairs. In the flux tube model, low-lying hybrids are expected to decay into one ground state $S$-wave meson and one excited $P$-wave meson, thus favoring decays such as $b_{1}(1235) \pi$ or $a_{2}(1320) \pi$ and disfavoring decays such as $\pi \eta$ or $\pi \rho$. If this selection rule holds it may explain the failure to uncover hybrid mesons to date since the favored decays involve complicated multi-pion final states. The current experimental evidence for exotic hybrids from $\pi^{-} p$ interactions and $\bar{p} p$ annihilations, while tantalizing, is weak and not without controversy. That evidence is reviewed in what follows.

Photon probes and exotic hybrid mesons. Phenomenological arguments indicate that the photon is a probe that should be particularly effective in producing exotic hybrids but data on photoproduction of light mesons are sparse indeed. The first excited transverse modes of the flux tube are degenerate and correspond to clockwise or counterclockwise rotations of the flux tube about the axis joining the quark and antiquark fixed in space with $J=1$. Linear combinations of these two modes are eigenstates of parity and lead to $J^{P C}=1^{+-}$and $J^{P C}=1^{-+}$for the excited flux tube. When these quantum numbers are combined with those of the $q \bar{q}$ with $\vec{L}=0$ and $\vec{S}=1$ (quark spins aligned) three of the six possible $J^{P C}$ have exotic combinations: $0^{+-}, 1^{-+}$and $2^{+-}$. In contrast, when the $q \bar{q}$ have $\vec{L}=0$ and $\vec{S}=0$ (spins anti-aligned), the resulting quantum numbers of the hybrid meson are not exotic. Pion probes are $q \bar{q}$ with quark spins anti-aligned. If we view one outcome of the scattering process as exciting the flux tube that binds the quarks in the probe, the suppression of exotic hybrids in $\pi$-induced reactions is not surprising - a spin flip of one of the quarks is required followed by the excitation of the flux tube. Because a photon can fluctuate into a virtual $q \bar{q}$ with quark spins aligned and an excited flux tube by, for example, diffractive scattering, exotic hybrids are expected to be more readily produced by photons. 
Identifying the spectrum of exotic hybrid mesons. The GlueX experiment will initially focus on exotic $J^{P C}$ hybrid mesons since these states cannot mix with conventional $q \bar{q}$ mesons. The identification of these states requires a partial wave analysis (PWA) of exclusive reactions. For a given reaction, e.g. $\gamma p \rightarrow X p$ where $X$ refers to a specific set of detected mesons $\left(\pi^{ \pm}, \pi^{0}, K^{ \pm}, \eta, \ldots\right)$ the decay angular distribution of final state particles is fitted to a finite set of partial waves that characterize the production of $X$ and its decay. The fitting procedure is carried out in bins of $X$ mass, and the net result is the mass dependence of individual partial waves and their relative phase differences. Thus states are identified by their Breit-Wigner line shapes and interferences with each other and well-established meson resonances. Consistency checks among different decay modes of purported states are important to validate results.

This procedure makes stringent demands on the detector to identify exclusive reactions by kinematic fitting. This requires excellent hermiticity, energy and momentum resolution, and particle identification. Excellent and well-known acceptance is required in the fitting procedure that is part of the PWA. The GlueX detector has been designed to meet these requirements. Optimization studies indicate that the ideal detector for a photon beam should be based on a solenoidal spectrometer magnet and that $9 \mathrm{GeV}$ photons are ideal for providing the required mass reach and resolution. Large statistics data sets are also required to enable the systematic studies needed to identify and understand the signals for exotic hybrid states. This requirement has set the design of the GlueX triggering and data acquisition system. In addition, the measurements depend on the unique ability of the CEBAF accelerator to provide an electron beam with both high enough energy and a duty factor close to one. Part of the studies leading up to the design of the GlueX detector involved double-blind tests in which a mix of partial waves corresponding to a various $J^{P C}$ states of various masses, including known states and hypothetical states with small cross sections relative to conventional mesons were used to generate simulated data. These Monte Carlo data underwent resolution and spatial smearing with simulated detector response. The reconstructed events were corrected for acceptance and then passed to a fitter. Based on statistics expected from one year of running at $10^{7}$ photons/s, we expect to reliably extract exotic signals that are as low as $5 \%$ of the signals from conventional meson states.

Linearly polarized photons are required to extract maximum information from the PWA including information on selecting production mechanisms and filtering quantum numbers of produced states. Linearly polarized photons will be produced by coherent bremsstrahlung from thin diamond crystals. Recent developments only now make possible production of wafers of sufficient quality and thin enough to make possible the required collimation of the photon beam for GlueX. The technique also requires the superb beam characteristics of the CEBAF electron beam (spot size and emittance) and a minimum energy of $12 \mathrm{GeV}$ to achieve the requisite degree of linearly polarization for $9 \mathrm{GeV}$ photons.

Spectroscopy of conventional mesons including strangeonium. The data that will be accumulated in the search for exotic hybrid mesons will also provide new information about normal $q \bar{q}$ mesons and, in particular, on the spectroscopy of strangeonium $-s \bar{s}$ mesons. The application of the PWA technique necessarily includes information about normal $q \bar{q}$ mesons, both established states and those yet to be discovered. This also applies to the identification of hybrid mesons with non-exotic quantum numbers that can mix with normal $q \bar{q}$ states. Thus, as part of the program of identifying hybrid mesons, the high statistics data sets will yield information on ground state $q \bar{q}$ mesons as well. 


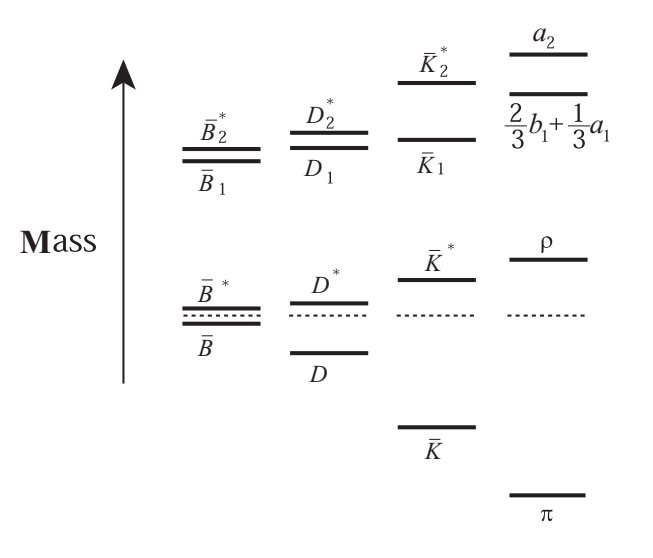

Q⿳亠口冋 States

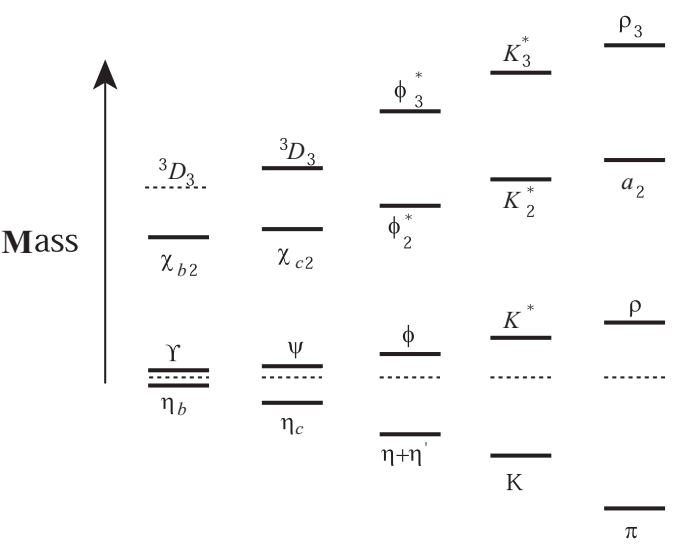

QQ States

Figure 2: The relative splittings of the $Q \bar{d}$ states and the $Q \bar{Q}$ states, both shown from the heaviest to the lightest. (Left) The relative splittings of the $Q \bar{d}$ states are shown to scale from the heaviest to the lightest with the center-of-gravity of the ground state multiplets aligned: $b \bar{d}, c \bar{d}, s \bar{d}$, and $u \bar{d} . \quad \bar{B}^{*}$ and $\bar{B}$ are the $J^{P}=1^{-}$and $0^{-}$"ground state" multiplet with light-degrees-of-freedom spin-parity $s_{\ell}^{\pi_{\ell}}=\frac{1}{2}^{+}$, while $\bar{B}_{2}^{*}$ and $\bar{B}_{1}$ with $J^{P}=2^{+}$and $1^{+}$are an excited heavy-quark spin multiplet with $s_{\ell}^{\pi_{\ell}}=\frac{3}{2}^{+}$[Is91]. (Right) The $Q \bar{Q}$ states are shown from the heaviest to the lightest: $b \bar{b}, c \bar{c}, s \bar{s}, s \bar{d}$, and $u \bar{d}$. Shown are the states in each sector with $J^{P C}=0^{-}, 1^{-}, 2^{+}$, and $3^{-}$; relative splittings are shown to scale with the center-of-gravity of the "ground states" $0^{-}$and $1^{-}$aligned.

The $s \bar{s}$ (strangeonium) spectrum will yield new information on the transition from heavy quarks to light quarks in QCD. The left half of Fig. 2 summarizes information about the spectra of $Q \bar{q}$ mesons for $\bar{q}$ a light $(\bar{d})$ quark and $Q=b, c, s$, and $u$. The rigorous results of Heavy Quark Effective Theory (HQET) should only be applicable for $Q=b, c$, but these data suggest that there is a remarkable similarity between the dynamics of "true" heavy-light systems and those where $Q=s$ or even $Q=u$ or $d$. It appears that the creation of the constituent quark mass through spontaneous chiral symmetry breaking is enough to boost any quark into the heavy-quark world, at least qualitatively. The right half of Fig. 2 shows heavy quarkonia ( $Q \bar{Q}$ systems) spectra starting from the heaviest $b \bar{b}$ system to the lightest. Even though there is no known rigorous explanation, there appears to be a similarity between the spectra of the heavy quarkonia (which have a wellunderstood quark-model-like connection to QCD) and light-quark systems.

All this suggests that more information about $s \bar{s}$ spectroscopy is needed. Given that the photon often fluctuates into an $s \bar{s}$ pair, a great deal of data will automatically be available from this sector as part of the planned exotic hybrid meson program. Strangeonium should decay into systems such as $\phi \eta, \phi \phi, K^{*} \bar{K}$ and $K^{*} \bar{K}^{*}$ - accessible to GlueX because of its excellent particle identification. Given that the intrinsic $s \bar{s}$ content of the proton is expected to be small, photoninitiated $s \bar{s}$ spectroscopy will strongly favor the production of diffractive-type $C=-1$ states. The exception will be channels where OZI-violating $t$-channel exchanges (like those of the $\eta-\eta^{\prime}$ system) can occur. These effects will result in an uneven population of the spectrum. The very high data rates anticipated should nevertheless lead to a data set of sufficient quality that even the weakly excited states will still be identifiable. 
The GlueX program will also be able to make a substantial contribution to baryon spectroscopy. The same characteristics (hermeticity, broad acceptance, and excellent particle ID) that were the focus of the detector design for meson spectroscopy are essential for baron spectroscopy. The higher energy of the Upgrade beams will extend baryon spectroscopy to include both higher excitation energies and more exotic decay modes, such as double-strangeness cascades, which are expected to have smaller widths and therefore be easier to see experimentally.

Summary. Advances in science are driven by advances in technology, and GlueX can now address the experimental challenge of mapping exotic mesons because of recent developments in producing thin diamond crystals (necessary for producing photon beams of requisite polarization), electronics (to achieve data-taking rates), and significant increases in computational power (needed to analyze large data sets). With the energy upgrade of the CEBAF accelerator, the final requirement to do this fundamental physics will have been met. In parallel, increased computational power will also enable LQCD calculations to yield the detailed prediction of the spectrum of exotic hybrid mesons whose validation will lie in the experimental information from the GlueX experiment. Nowhere else are accelerator and experiment so uniquely poised to exploit the discovery potential needed to address an important and fundamental question in physics - the nature of confinement in QCD.

\section{B The Fundamental Structure of the Nuclear Building Blocks}

Nucleons (i.e., protons and neutrons) are the basic building blocks of atomic nuclei. Their interactions determine the fundamental properties of nuclei, which, in turn, define the chemical elements and their properties. They also determine the dynamics of nuclear reactions that fuel the Sun and govern the evolution of the early Universe. The nucleons exhibit a complex internal structure of their own, which is inseparably linked with their interactions. It is this fact which accounts for the fascinating richness of nuclear phenomena, and which makes the physics of strong interactions a uniquely challenging problem. Unraveling the internal structure of the nucleon is the key to understanding phenomena at the nuclear and astrophysical level.

The structure of the nucleon is governed by the fundamental theory of strong interactions, quantum chromodynamics (QCD). It describes the structure in terms of point-like elementary constituents, quarks and gluons, whose interactions follow from a quantum field theory. The quarks and gluons are directly observable only when probing the nucleon at distances significantly smaller than $1 \mathrm{fm}=10^{-15} \mathrm{~m}$, i.e., in reactions at high momentum transfers. At distances of the order of $1 \mathrm{fm}$, the interactions between the quarks give rise to phenomena that qualitatively change the character of the dynamics - the confinement of quarks (absence of free quarks), and the spontaneous breaking of chiral symmetry (generation of dynamical quark masses). These phenomena make the actual calculation of nucleon properties in QCD an exceedingly complex problem, which is addressed either by large-scale computer simulations or by dynamical models using effective degrees of freedom. Our understanding of the fundamental quark and gluon structure of the nucleon based on quantum chromodynamics is still at a very preliminary stage compared to our understanding of atomic structure based on quantum electrodynamics [Th01].

Electron scattering experiments have played a crucial role in exploring the internal structure of the nucleon. The first direct evidence for the extended nature of the proton came from measurements of the form factors of $e N$ elastic scattering (Hofstadter, Nobel Prize). The form factors are 
directly related to the spatial distributions of the electric charge and current inside the nucleon. Another milestone was the discovery of scaling in inelastic $e N$ scattering at large momentum transfer, $Q^{2}$ (Friedman, Kendall, and Taylor, Nobel Prize). This provided experimental confirmation of the existence of quarks, and established the phenomenon of asymptotic freedom implied by QCD (Gross, Politzer, and Wilczek, Nobel Prize). Such inelastic scattering experiments measure the momentum distribution of quarks in a fast-moving nucleon along the direction of motion ("parton distributions"), and thus provide information of completely different nature from that contained in the form factors. Later deep inelastic scattering experiments at high energies confirmed the QCD predictions that the $Q^{2}$ dependence of the parton distributions is associated with gluon radiation.

While these experiments have shaped our basic understanding of strong interactions, they really offer only a first glimpse of the fundamental quark and gluon structure of the nucleon. Recent advances in experimental technology and theoretical formulation make it possible to answer much more detailed questions:

- 3D quark/gluon imaging of the nucleon: Can we understand the nucleon as an extended object in terms of quark and gluon degrees of freedom? The elastic form factors (spatial charge distributions) and parton distributions (momentum distribution of quarks/gluons in a fastmoving nucleon) have been shown to be special cases of a more general concept, the so-called "generalized parton distributions" (GPD's). These new observables describe the distribution of quarks and gluons in terms of both their longitudinal momentum and their transverse position, and thus for the first time provide us with "3D images" of the quark and gluon structure of the nucleon. They can be measured directly in exclusive $e N$ scattering processes at large $Q^{2}$ in which the nucleon is observed intact in the final state.

- Valence quark structure: What are the properties of the nucleon in the regime where the three valence quarks dominate? The high- $x$ region is a unique and vital testing ground for theoretical models of the nucleon, which at low energies are formulated in terms of valence quark degrees of freedom. The $12 \mathrm{GeV}$ Upgrade will be the first facility in over 35 years of studies of deep inelastic scattering with the duty factor, luminosity and reach in $Q^{2}$ required to explore with precision the spin and flavor dependence of the parton distribution functions in this fundamentally important regime.

- Spin structure of the nucleon: How does the nucleon spin arise as a result of the spins of the elementary quarks and gluons and their orbital motion? Semi-inclusive scattering experiments with polarized beams and targets allow us to determine how the quark spin is correlated with the quark momentum, flavor and charge (quark/antiquark). This information will make possible a much more detailed understanding of the binding of quarks and gluons in the nucleon.

- The parton-hadron transition: How does the "partonic" (quark/gluon) structure of the nucleon relate to its hadronic excitation spectrum? Inelastic scattering experiments in the resonance region allow us to study how the coherent excitation of nucleon resonances turns into the incoherent scattering off pointlike quarks with increasing momentum transfer. This fascinating property, called quark-hadron duality, is the clue to understanding the role of confinement and chiral symmetry breaking in the structure of the nucleon.

The $12 \mathrm{GeV}$ Upgrade of CEBAF offers a unique possibility to answer these fundamental questions. It provides the necessary electron energy to reach momentum transfers at which the elementary 


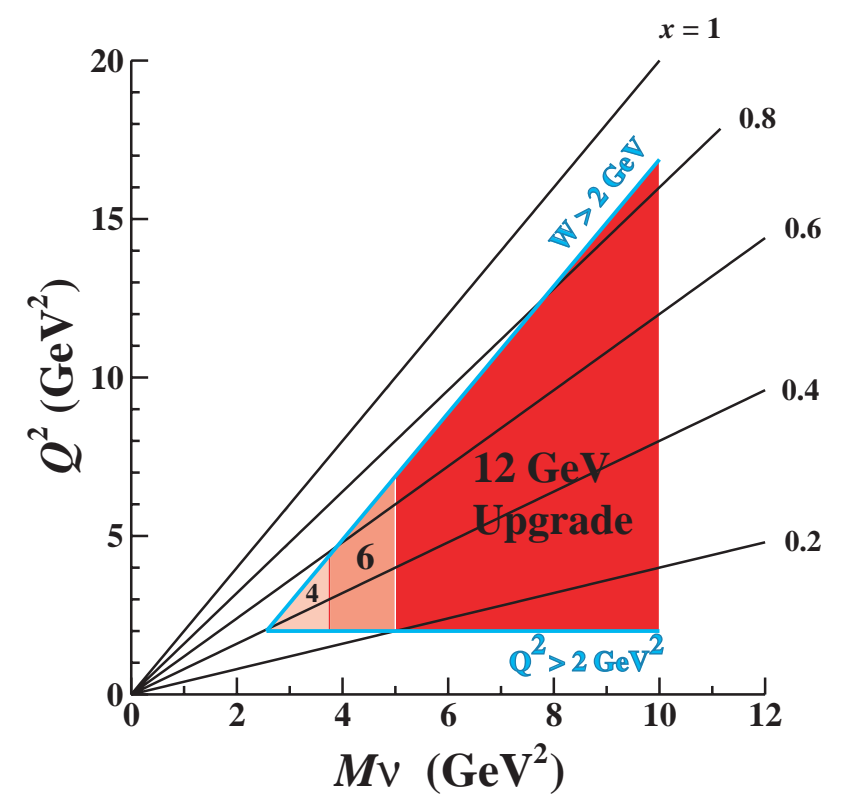

Figure 3: The kinematic coverage in momentum transfer, $Q^{2}$, as a function of the mass of $(M)$ and energy transferred to $(\nu)$ the target by the electron. This plot conveniently identifies the deep inelastic scattering regime as a triangle bounded on the right by the beam energy. The fraction of the proton's momentum carried by the struck quark (given by the Bjorken variable, $x$ ) appears as radial lines on the plot. From this figure we can infer the range of $x$ and $Q^{2}$ accessible to deepinelastic $e N$ scattering with the $12 \mathrm{GeV}$ Upgrade of CEBAF and the large enhancement relative to the original CEBAF energy of $4 \mathrm{GeV}$.

QCD degrees-of-freedom become visible. Kinematically this regime has been accessible before, for example at SLAC. An essential feature of the Upgrade is that it provides not only the energy but also the luminosity and polarization required to study low-rate processes such as deep-inelastic scattering at large $x$, exclusive and semi-inclusive reactions, and measurements of polarization asymmetries. As an example of the new reach afforded by the Upgrade, Fig. 3 shows the kinematic coverage for measurements in the deep-inelastic region. The accelerator design will be complemented by a set of detectors specifically designed to cover this kinematic region. Together, they will support a broad range of inclusive, semi-inclusive and exclusive measurements that will produce a comprehensive picture of the quark and gluon structure of the nucleon. No other facility presently available or planned offers comparable capabilities for carrying out the envisaged research program.

3D quark/gluon imaging of the nucleon. It has long been known that the form factors of elastic $e N$ scattering at low $Q^{2}$ can be represented as the Fourier transform of the spatial electric charge and current density in the Breit frame. Recently it was realized that this idea can be extended to much more general distributions of quarks and gluons in QCD. These are the so-called generalized parton distributions (GPD's), which unite the concept of the elastic form factor with that of the quark and antiquark distributions measured in inclusive $e N$ scattering at large $Q^{2}$ [Co97, Ji97, Ra97]; see Refs. [Di03, Be05] for recent reviews. The GPD's describe the amplitudes for a fast-moving nucleon to emit and absorb a quark (or antiquark). They depend on the longitudinal momentum fractions of the quark, as well as on the invariant momentum transfer 


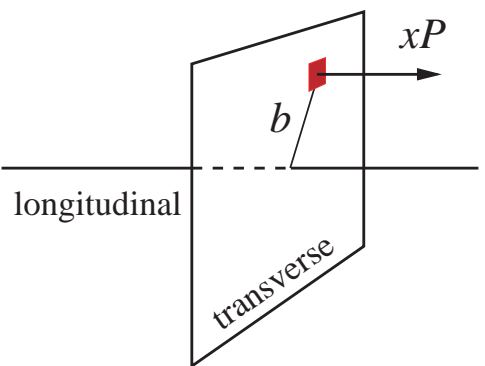

(a)

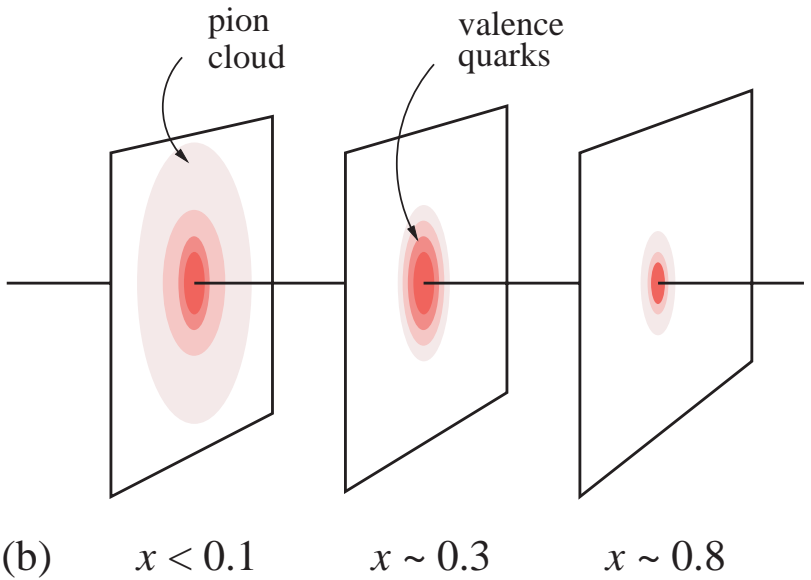

(b)

$x<0.1$

$x \sim 0.3$

$x \sim 0.8$

Figure 4: Nucleon tomography: (a) The Fourier transform of the GPD describes the simultaneous distribution of quarks with respect to longitudinal momentum, $x P$, and transverse position, $b$, in the infinite momentum frame. (b) It produces a 1+2-dimensional "tomographic" image of the quark structure of the nucleon [Bu03], which, for example, allows one to clearly identify the contribution from the valence quarks $(x \geq 0.3$, moderate $b)$ and the pion cloud $\left(x \leq 0.1, b \sim 1 / M_{\pi}\right)$ [St03a].

to the nucleon, $t$. In the diagonal case (zero momentum transfer) they reproduce the "usual" quark densities measured in inclusive $e N$ scattering. On the other hand, when integrated over the quark momentum fraction, the GPD's reproduce the elastic nucleon form factors.

The Fourier transform of the GPD's with respect to the momentum transfer to the nucleon describes the simultaneous distribution of quarks and gluons with respect to longitudinal momentum fraction and transverse position ("impact parameter-dependent parton densities") in the infinite momentum frame (see Fig. 4 [Bu03, Di02]). These functions provide a " $1+2$ "-dimensional representation of the parton structure of the nucleon, which offers unprecedented possibilities for visualizing the nucleon as an extended object, and provides a new arena for testing dynamical models of its structure. The transverse spatial distribution of partons is also a crucial ingredient in modeling high-energy proton-(anti)proton collisions with hard QCD processes, which are studied at the Fermilab Tevatron and the CERN LHC [Fr04].

The new representation also naturally lends itself to the discussion of polarization phenomena. For example, the Fourier transform of the Pauli form factor-type GPD can be interpreted as the distortion of the longitudinal motion of the quarks induced by a transverse polarization of the nucleon [Bu02]. The second moments of the Dirac and Pauli form-factor type GPD's contain the information about the total (spin plus orbital) quark contribution to the nucleon spin, $J^{q}=$ $\frac{1}{2} \Delta \Sigma+L^{q}[\mathrm{Ji} 97]$. A measurement of this quantity, combined with existing data on $\Delta \Sigma$ from inclusive deep-inelastic scattering, will make it possible to determine the quark orbital angular momentum, $L^{q}$. The GPD's also contain information about the forces acting on the quarks inside the nucleon [Po03].

More generally, the GPD's can be related to the quantum phase-space (Wigner) distributions of quarks and gluons in the nucleon [Ji03, Be03]. A phase-space function represents a correlated momentum and coordinate distribution, and contains much more information than the momentum or coordinate space distribution alone. Phase space distributions have been widely used in many 


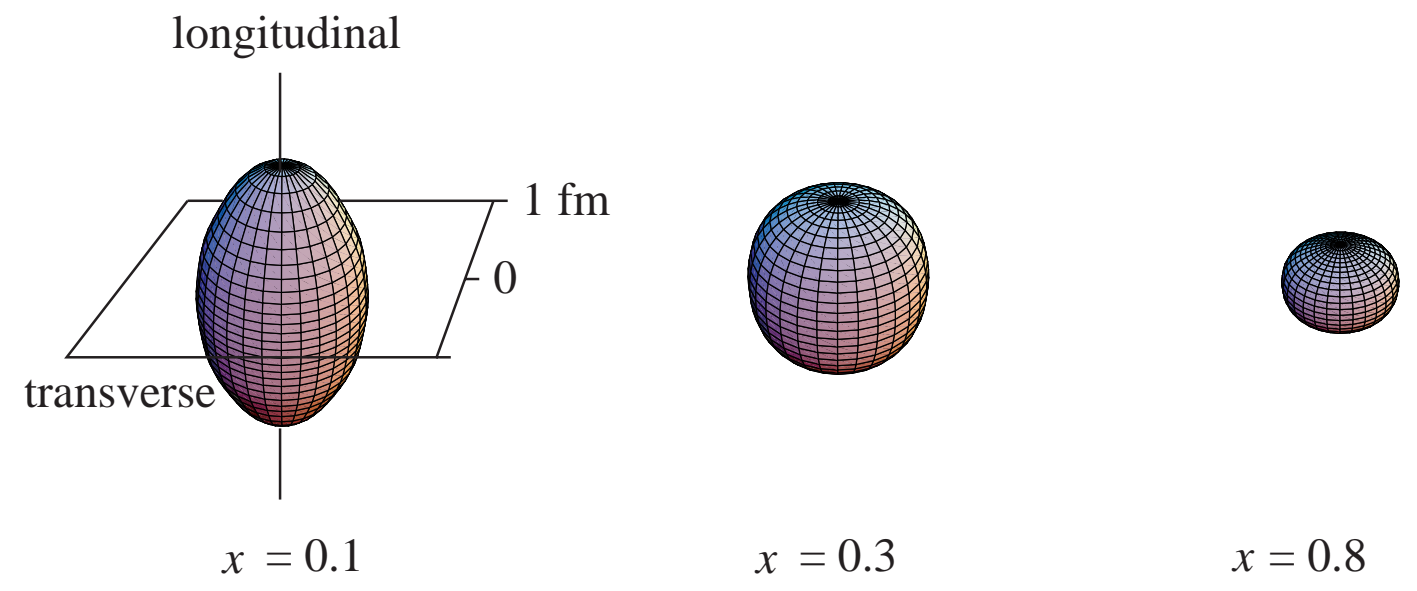

Figure 5: "3D images" of the nucleon, as obtained from a model phase space distribution incorporating phenomenological information about GPD's [Be03]. Shown are constant-density surfaces of the spatial distribution of $u$-quarks, for three values of the momentum fraction, $x$. In the valence quark region $(x \geq 0.1)$ the nucleon has a spherical shape. The size shrinks at larger $x$. At small $x$, the quarks spread out in the longitudinal direction, and the shape becomes cigar-like.

different areas of physics and technology, but have so far not been studied systematically for subatomic systems. An example of the power of phase space distributions for visualizing the partonic structure of the nucleon is given in Fig. 5. It shows the spatial distribution of $u$-quarks in the proton for different values of the momentum fraction, $x$, as reconstructed from a GPD model fitted to available form factor and parton distribution data [Be03]. By accumulating more and more information about the GPD's from a variety of measurements, we can gradually refine such images, and thus reconstruct the 3D quark-gluon structure of the proton with unprecedented detail.

The mapping of the generalized parton distributions and the construction of a comprehensive $3 \mathrm{D}$ image of the quark structure of the nucleon are prime objectives of the $12 \mathrm{GeV}$ Upgrade. This will be an extensive program rather than a single experiment, involving the measurement of a variety of channels (inclusive and exclusive) over a broad kinematic range. The GPD's incorporate the full information about the longitudinal momentum distribution of the quarks obtained from inclusive $e N$ scattering. Measurements of the nucleon elastic form factors will provide constraints on the first moments of the GPD's and their $t$-dependence. Finally, measurements of exclusive reactions in $e N$ scattering at large $Q^{2}$, in particular deeply-virtual Compton scattering and meson production, will allow us to map the $x$ - and $t$-dependence of the GPD's in detail.

Nucleon elastic form factors. Measurements of the nucleon elastic form factors map the charge and current distributions in the nucleon. In spite of a long history of such experiments, of the four form factors for elastic $e N$ scattering (electric and magnetic, proton and neutron) only the magnetic form factor of the proton, $G_{M}^{p}$, has been measured up to high $Q^{2}\left(\sim 30 \mathrm{GeV}^{2}\right)$ with relatively good accuracy. Recent JLab results show the potential for discovery with increasing $Q^{2}$, see Fig. 6. Contrary to long-held beliefs, the ratio $F_{2}^{p} / F_{1}^{p}$ is still far from the $1 / Q^{2}$ behavior implied by perturbative QCD up to $Q^{2} \approx 6 \mathrm{GeV}^{2}$. This demonstrates that the charge and magnetization distributions in the proton are quite different, and points to the importance of higher orbital angular momentum components in the proton wavefunctions. With the $12 \mathrm{GeV}$ Upgrade, the ratio $F_{2}^{p} / F_{1}^{p}$ 


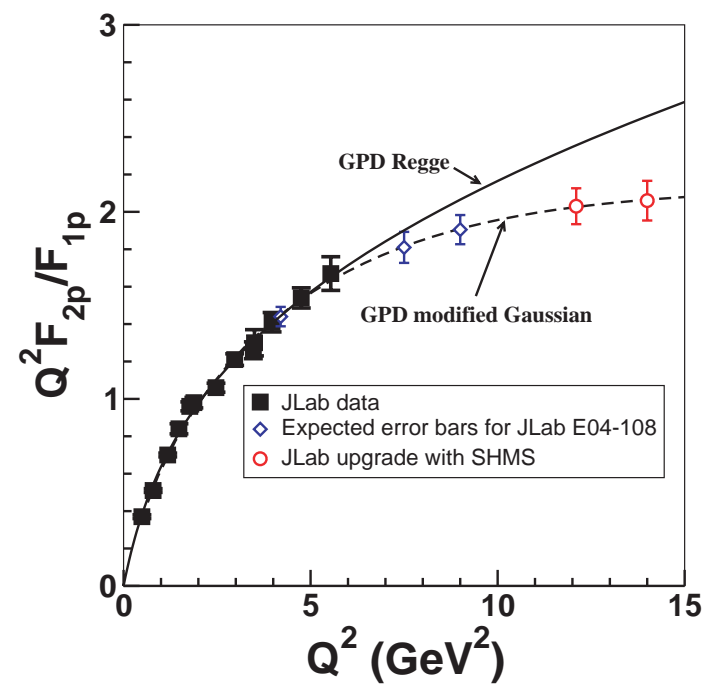

Figure 6: The JLab $6 \mathrm{GeV}$ data on the ratio of proton form factors, $F_{2}^{p} / F_{1}^{p}$, and the projected measurements at $12 \mathrm{GeV}$. Perturbative QCD implies that $F_{2}^{p} / F_{1}^{p} \sim 1 / Q^{2}$ for $Q^{2} \rightarrow \infty$. The graph illustrates the potential of form factor measurements at $12 \mathrm{GeV}$ to discriminate between GPD models. Shown are the predictions of a Regge-based GPD model [Gu04] and a model based on soft Gaussian nucleon wavefunctions modified by a short-range interaction [St03d].

could be measured up to $14 \mathrm{GeV}^{2}$, probing the proton's structure at distances as small as $0.1 \mathrm{fm}$. Knowledge of neutron form factors at high $Q^{2}$ is equally important. With the $12 \mathrm{GeV}$ Upgrade, the neutron magnetic form factor could be measured up to about $14 \mathrm{GeV}^{2}$, and its electric form factor up to $8 \mathrm{GeV}^{2}$.

The $12 \mathrm{GeV}$ form factor measurements will provide crucial constraints on the first moments of the GPD's and their $t$-dependence. Fig. 6 demonstrates the power of form factor measurements to discriminate between GPD models. An equally important consideration is that form factors can be measured up to high $|t|$ (= $Q^{2}$ for elastic scattering), while direct measurements of the GPD's in exclusive processes are restricted to $|t| \leq 1 \mathrm{GeV}^{2}$. Thus, form factor measurements will continue to provide our only source of information about the quark distributions at small transverse distance scales.

GPD's in deeply-virtual Compton scattering. GPD's can be measured in certain exclusive processes in $e N$ scattering, namely Deeply Virtual Compton Scattering (DVCS) and Deeply Virtual Meson Production (DVMP). At large $Q^{2}$ and scattering energy, these processes are dominated by the scattering from a single quark or antiquark, whose emission and absorption by the nucleon is described by the GPD's, see Fig. 7. The amplitude of the "hard" scattering process at the quark level can be calculated in perturbative QCD (factorization). The measured cross sections and spin asymmetries can then be used to extract the "soft" information about the nucleon contained in the GPD's.

In the reaction $e N \rightarrow e N \gamma$ at JLab energies, photons are produced not only via DVCS from the nucleon, but also (and even more copiously) via the electromagnetic Bethe-Heitler (BH) process. The two processes interfere, and the $\mathrm{BH}$ term, which is completely determined by the well-known 
a)

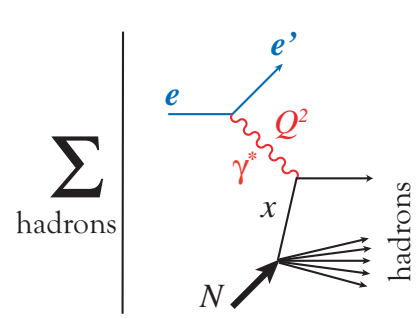

Inclusive Deep-Inelastic Scattering (DIS)
2

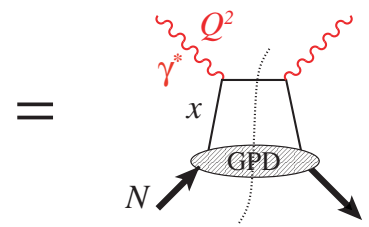

Forward Compton Scattering Amplitude

b)

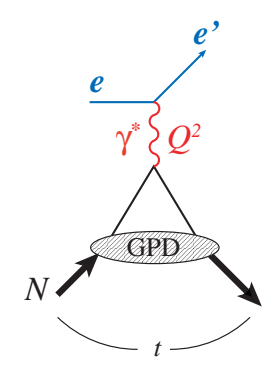

Elastic form factors c)

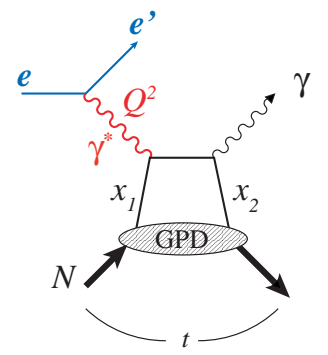

Deeply Virtual Compton Scattering (DVCS) d)

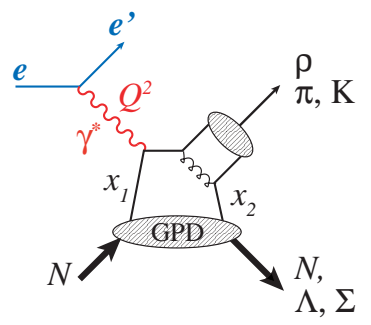

Deeply Virtual Meson Production (DVMP)

Figure 7: Overview of reactions in $e N$ scattering probing the generalized parton distributions. (a) Inclusive deep-inelastic scattering probes the GPD's in the "diagonal" case (zero momentum transfer to the nucleon), where they coincide with the usual parton densities in the nucleon. (b) The elastic form factors of the nucleon constrain the moments ( $x$-integrals) of the GPD's at non-zero momentum transfer, t. (c) Deeply-virtual Compton Scattering (DVCS) probes the GPD's at nonzero $t$ and longitudinal momentum transfer, $x_{2} \neq x_{1}$. The Compton process takes place in the reaction with a single quark or antiquark, whose emission and absorption by the nucleon is described by the GPD's. (d) GPD's in deeply-virtual meson production. Different mesons $(\rho, \pi, K)$ probe different spin-flavor components of the GPD's. 

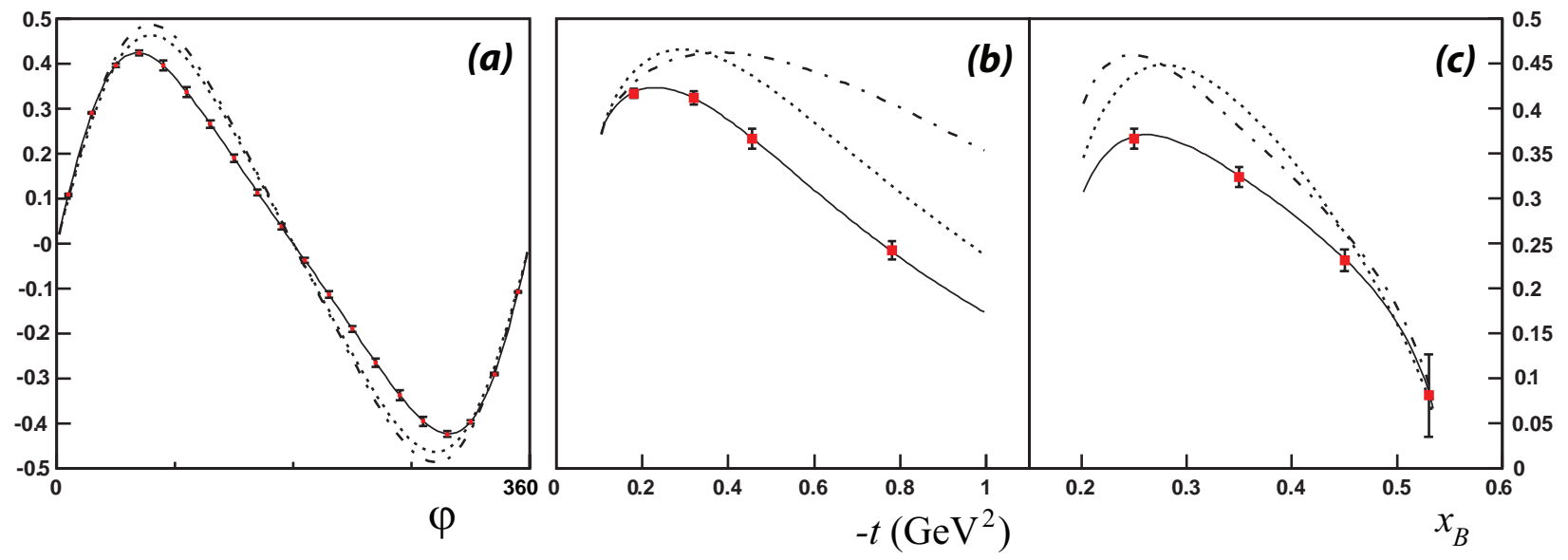

Figure 8: Projected measurements DVCS via $e p \rightarrow e p \gamma$ at $12 \mathrm{GeV}$. In each figure the full line correponds to a model with a Regge-type $t$-dependence and D-term. The dotted line includes the Regge-type $t$-dependence but has no D-term. The dash-dotted line has the D-term but the $t$ dependence only comes from form factors. (a) Beam spin asymmetry (BSA) as a function of $\varphi$ for $<x_{B}>=0.2,<Q 2>=3.3 \mathrm{GeV}^{2}$ and $<-t>=0.45 \mathrm{GeV}^{2}$. (b) BSA as a function of $-t$ for $<x_{B}>=0.2,<Q 2>=3.3 \mathrm{GeV}^{2}$ and $\varphi=90^{\circ}$. (c) BSA as a function of $x_{B}$ for $t=0.45 \mathrm{GeV}^{2}$, $<Q 2>=3.3 \mathrm{GeV}^{2}$ and $\varphi=90^{\circ}$. [Gu04, Va99].

elastic form factors, amplifies the much smaller DVCS term to comfortably measurable levels, making it possible to extract information about the GPD's. DVCS is an established tool for GPD studies, with experimental results reported by CLAS [St01] at JLab, HERMES [Ai01], and the HERA experiments H1 [Ad01] and ZEUS [Sa03]. The existing data are well described by a consistent GPD analysis in leading order (LO) and next to leading order (NLO) QCD [Fr01], supporting the feasibility of experiments aimed at determining the GPD's via DVCS even at relatively low $Q^{2}$. With the beam energy of $6 \mathrm{GeV}$ these experiments are limited to a small part of the relevant range of kinematic variables. The $12 \mathrm{GeV}$ Upgrade will allow us to perform DVCS measurements with a much broader kinematic coverage in $Q^{2}, x$, and $t$ (see Fig. 3).

Measurements of the beam spin asymmetry in $e N \rightarrow e N \gamma$ allow for the extraction of GPD's at fixed values of the quark momentum fractions, while measurements of the cross section determine integrals of the GPD's over the momentum fractions. As an example, Fig. 8 illustrates the precision and kinematic coverage expected for measurements of the beam asymmetry with the $12 \mathrm{GeV}$ Upgrade. Measurements of target spin asymmetries ( $L$ and $T$ ) will allow us to isolate individual GPD's, corresponding to definite nucleon and quark helicity states. Finally, measurements of the $t^{-}$ dependence will allow us to obtain information about the transverse spatial distribution of quarks.

A much more detailed mapping of the $x$-dependence of the GPD's than in DVCS is possible in the electroproduction of virtual photons, which decay into $e^{+} e^{-}$pairs (Double Deeply-virtual 


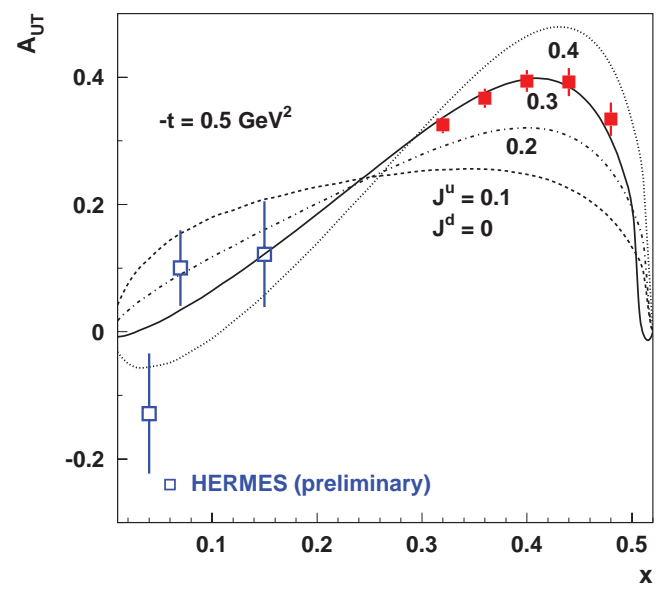

Figure 9: Sensitivity of the transverse target spin asymmetry in deeply-virtual $\rho^{0}$ production to the total $u$-quark angular momentum in the proton, $J^{u}$. The curves were obtained using a GPD parameterization with $J^{u}$ as a free parameter [Go01]. The red data points illustrate the precision of the projected measurements at $12 \mathrm{GeV}$; the blue data points are the preliminary results reported by the HERMES experiment [Dr05]

Compton scattering, DDVCS) [Gu03, Be03a]. Because of the additional electromagnetic interaction the cross section is significantly suppressed as compared to DVCS. Nevertheless, the feasibility of DDVCS measurements has been demonstrated in a pioneering study at JLab with the $6 \mathrm{GeV}$ beam.

GPD's in deeply-virtual meson production and other processes. Deeply-virtual meson production (DVMP) will be a valuable tool for separating different spin and flavor components of the GPD's. Vector meson production $(\rho, \omega)$ isolates the spin-independent GPD's and helps to separate $u$ and $d$ quark contributions. In particular, the transverse target spin asymmetry in $\rho^{0}$ production probes the Pauli form factor-type GPD, which is needed to extract the total quark angular momentum in the nucleon, $J^{q}$. Figure 9 shows the sensitivity of this observable to the $u$-quark angular momentum, and illustrates the statistical precision of the planned measurements at $12 \mathrm{GeV}$. Pseudoscalar meson production $\left(\pi^{0}, \eta\right)$ accesses the spin-dependent GPD's. Kaon production, which probes the $N \rightarrow \Sigma, \Lambda$ "transition GPD's", provides information about strangeness in the nucleon and the flavor structure of octet baryons [Go01]. However, while in DVCS the data can be interpreted directly within the GPD formalism, in DVMP the inclusion of higher-twist corrections, based on theoretical models, will be an essential part of the analysis.

The GPD formalism can also be used to "connect" other measurements of nucleon structure in a unified framework. Wide-angle real Compton scattering, which is dominated by the same partonic reaction mechanism as DVCS, probes certain $1 / x$-weighted moments of the GPD's [Ra98, Di99, Hu02], and can provide critical information on the parton structure of the nucleon at small distance scales. The gluon GPD structure of the nucleon can be probed using $J / \psi$ photoproduction near threshold [Br01, Fr02]. In particular, such measurements can determine the transverse size of the gluon distribution in the nucleon at large $x$, and provide information about the the dynamical origin of the non-perturbative glue in the nucleon. 


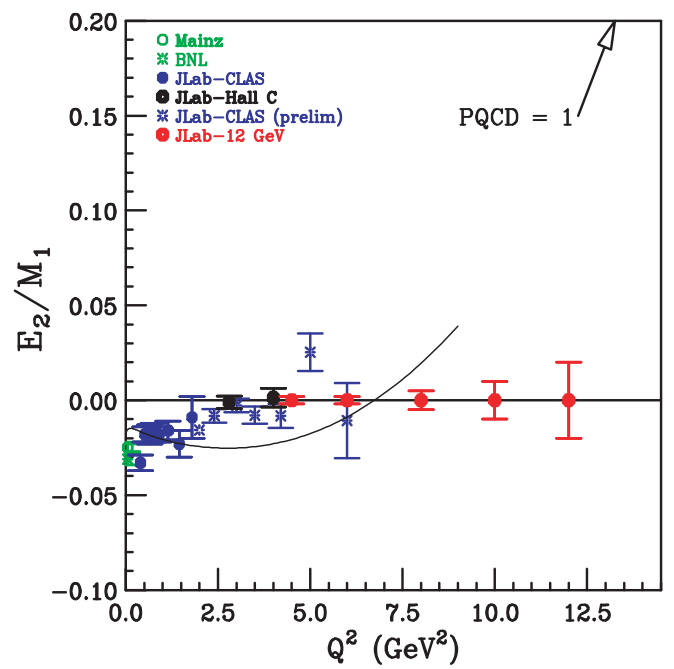

Figure 10: Projected measurements of the $E_{2} / M_{1}$ ratio for the $N \rightarrow \Delta$ transition form factors at $12 \mathrm{GeV}$. The curve represents the pion cloud model of Ref.[Ka99]. Perturbative QCD implies $E_{2} / M_{1} \rightarrow 1$ due to helicity conservation.

Resonance transition form factors and GPD's. While the elastic (ground state) form factors tell us about the distribution of electric charge and magnetization in the nucleon, the form factors of the electromagnetic transitions of the nucleon to its excited states provide information on how the quarks move in response to external electric and magnetic fields. The transition form factors are particularly sensitive to the angular momentum structure of the nucleon and resonance wave functions. A major unresolved issue is the determination of the momentum transfers at which these form factors begin to exhibit the scaling behavior implied by the pQCD hard scattering mechanism. As an example, Fig 10 shows the $E_{2} / M_{1}$ ratio of the $N \rightarrow \Delta$ transition. Perturbative QCD predicts that $E_{2} / M_{1} \rightarrow 1$ for $Q^{2} \rightarrow \infty$ due to helicity conservation. Existing data at low $Q^{2}$ show a ratio near zero. With the $12 \mathrm{GeV}$ Upgrade, $E_{2} / M_{1}$ will be measured up to $12 \mathrm{GeV}^{2}$, definitively answering the question of the relevance of the pQCD mechanism. Additional, more detailed information about the resonance structure can be obtained from measurements of the "resonance transition GPD's" [Fr99a]. Due to the much richer structure of the transition operator, DVCS can probe resonance excitation with quantum numbers not accessible in usual photo/electroproduction experiments.

The charged pion form factor. The electromagnetic form factor of the charged pion is of fundamental importance in hadronic physics. Due to the simple $q \bar{q}$ valence structure of the pion, the asymptotic behavior implied by pQCD [Ef80, Br79] is expected to set in at much lower values of $Q^{2}$ than in the nucleon case. Dynamical models of the pion suggest a significant role of non-perturbative effects in the form factor up to $Q^{2} \sim$ few $\mathrm{GeV}^{2}$. Present data cannot discriminate between the different theoretical estimates of the transition between non-perturbative and perturbative behavior (see Fig. 11). With the $12 \mathrm{GeV}$ Upgrade, the pion form factor will be measured accurately up to momentum transfers of $6 \mathrm{GeV}^{2}$. These measurements would provide precise tests of perturbative and non-perturbative calculations of pion structure in this $Q^{2}$ range, providing a definitive answer to a long-standing question in QCD. 


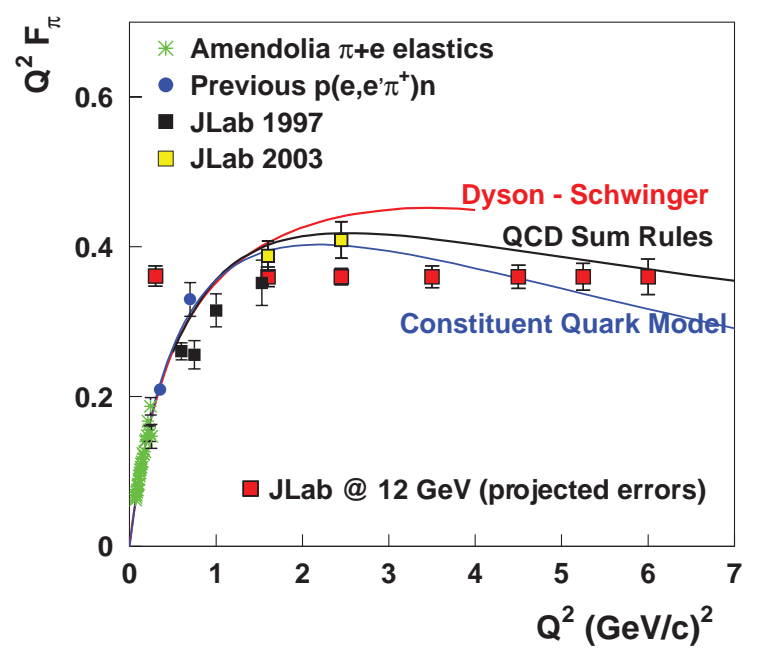

Figure 11: Projected measurements of the pion electromagnetic form factor, $F_{\pi}\left(Q^{2}\right)$, made possible by the proposed $12 \mathrm{GeV}$ Upgrade. Also shown are various model predictions for its behavior in the region $Q^{2} \sim$ few $\mathrm{GeV}^{2}$. Perturbative $\mathrm{QCD}$ predicts $F_{\pi} \sim 1 / Q^{2}$ for $Q^{2} \rightarrow \infty$.

Valence quark structure. One of the most fundamental properties of the nucleon is the structure of its valence quark distributions. Valence quarks are the irreducible kernel of each hadron, responsible for its charge, baryon number and other macroscopic quantum numbers. In deepinelastic scattering at average values of $x$, the valence quarks are "dressed" by quark-antiquark pairs produced by non-perturbative effects at large distance scales $(\sim 1 \mathrm{fm})$, as well as by gluon bremsstrahlung at short distances. At higher $x$ values these $q \bar{q}$ contributions drop away, and the physics of the valence quarks is cleanly exposed [Is99].

While deep inelastic scattering and other experiments have provided a detailed map of the nucleon's quark distributions at average $(\sim 0.3)$ and small values of $x$, there has never been an experimental facility capable of accurately measuring the cross sections throughout the "deep valence region" $(x>0.5)$ where the three basic valence quarks of the proton and neutron dominate the wavefunction. This represents a glaring gap in our knowledge of nucleon structure, especially since there are qualitatively different predictions for the quark spin and flavor distributions in the $x \rightarrow 1$ limit. The $12 \mathrm{GeV}$ Upgrade will for the first time provide the necessary combination of high beam intensity and reach in $Q^{2}$ to allow us to map out the valence quark distributions at large $x$ with high precision. These measurements will have a profound impact on our understanding of the structure of the proton and neutron. They will also provide crucial input for calculating cross sections for hard processes in high-energy hadron-hadron colliders such as the LHC, in searches for the Higgs boson or for physics beyond the Standard Model.

Valence quark spin distributions at large $x$. The $12 \mathrm{GeV}$ Upgrade will allow for measurements of inclusive spin structure functions at large $x$ with unprecedented precision. As an example, Fig. 12 shows the neutron polarization asymmetry, $A_{1}^{n}$, which is determined by a ratio of spin-dependent to spin-averaged quark distributions. Most dynamical models predict that in the limit where a single valence up or down quark carries all of the nucleon's momentum $(x \rightarrow 1)$, it will also carry all of the spin polarization (i.e., $A_{1}^{n} \rightarrow 1$ as $x \rightarrow 1$ ). Existing data on $A_{1}^{n}$ end before reaching the region of valence quark dominance, and show no sign of making the predicted 


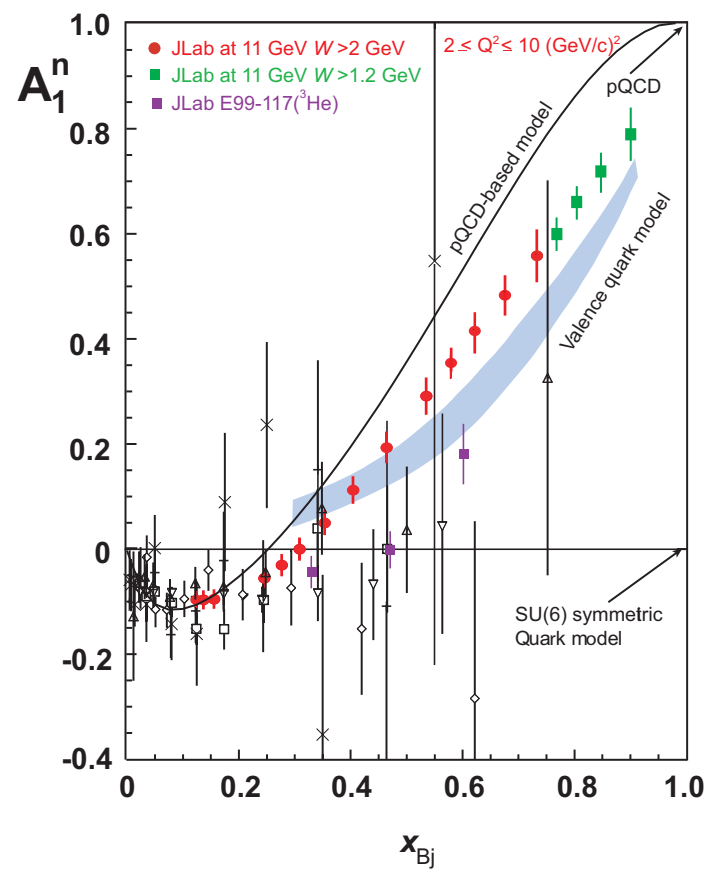

Figure 12: Projected measurement of the neutron polarization asymmetry, $A_{1}^{n}$, with the $12 \mathrm{GeV}$ Upgrade. The shaded band represents the range of predictions of valence quark models; the solid line is the prediction of a pQCD-based quark model.

dramatic transition $A_{1}^{n} \rightarrow 1$ (recent data from the JLab Hall A experiment E99-117 [Zh03a, Zh04] show the first hint of a possible upturn at the largest $x$ value). There is a similar lack of data on other deep inelastic scattering observables in this region.

Flavor structure of valence quarks at large $x$. Even in unpolarized deep-inelastic scattering, where the available data are best, there are long-standing unresolved issues. One example is the ratio of down to up quarks in the proton, $d(x) / u(x)$, whose large- $x$ behavior is intimately related to the fact that the proton and neutron, and not the $\Delta$, are the stable building blocks of nuclei [Cl88]. This ratio requires measurement of the neutron as well as the proton structure function. Information about the neutron has to be extracted from deuterium data, and is difficult to disentangle from nuclear effects (binding of $p$ and $n$ ) at large $x$ [Wh92, Me96]. Figure 13 shows the precision with which this fundamental ratio can be measured with the $12 \mathrm{GeV}$ Upgrade. The proposed experiment will utilize a novel technique, currently being pioneered at JLab; detection of the slowly recoiling proton spectator will "tag" scattering events on a nearly on-shell neutron in a deuteron target [Fr88, Me97]. An independent measurement of $d(x) / u(x)$ can be made by exploiting the mirror symmetry of $A=3$ nuclei in simultaneous measurements with ${ }^{3} \mathrm{He}$ and ${ }^{3} \mathrm{H}$ targets [AF00, Pa01, Sa01, Af03]. Both methods are designed to largely eliminate the nuclear corrections, thereby permitting the $d / u$ ratio to be extracted with unprecedented precision.

Spin structure of the nucleon. The precise way in which the spin of the nucleon is distributed among its quark and gluon constituents is one of the most fundamental questions that can be addressed in nonperturbative QCD. Starting from the famous "spin crisis" of the European Muon 


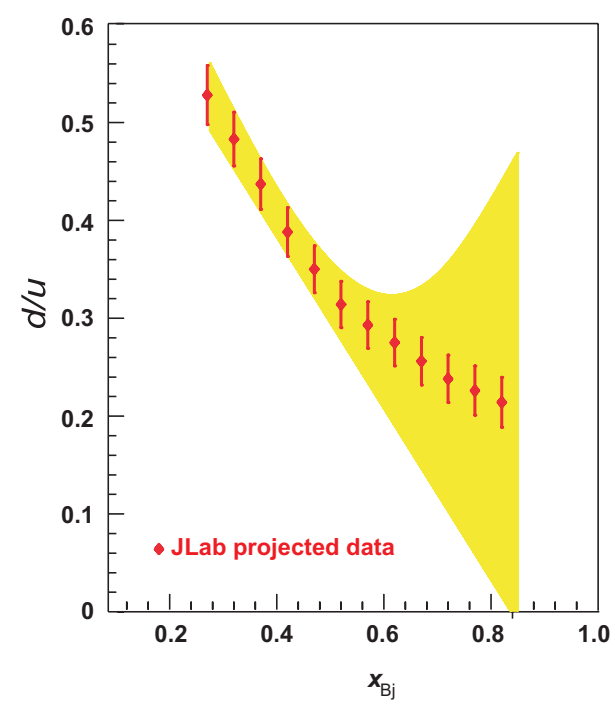

Figure 13: Projected measurement of the ratio of $d$ - and $u$-quark momentum distributions, $d(x) / u(x)$, at large $x$, made possible by the $12 \mathrm{GeV}$ Upgrade. The shaded band represents the uncertainty in existing measurements due to nuclear Fermi motion effects.

Collaboration, most of the experiments so far have focused on measuring the total quark and gluon contribution to the nucleon spin in inclusive deep-inelastic scattering, initiating numerous theoretical investigations of the role of gluon topology and the $U(1)$ axial anomaly in hadron structure [Ba02, An95]. Following on these results, in recent years the focus has been moving to the investigation of specific aspects of the nucleon spin, such as the flavor asymmetries of sea quark distributions and quark transverse spin (transversity) distributions.

Properties such as these are of particular interest, as they are free of contributions involving the axial anomaly, which affect the flavor-singlet quark spin distributions. They can therefore be related more directly to conventional models of nucleon structure. For example, the non-relativistic quark model, the meson cloud model, and the chiral quark-soliton model, make very different predictions for the flavor asymmetry of the polarized sea quark distributions. The difference between transversely and longitudinally polarized quark distribution (transversity and helicity) is a measure of the relativistic nature of the motion of quarks in the nucleon - see Ref. [Ba01] for a review. The quark transversity distributions are also intimately related to the chiral properties of QCD, being entirely due to chirally odd (helicity-flipping) effective interactions induced by the spontaneous breaking of chiral symmetry in QCD.

The mapping of the flavor dependence of polarized valence and sea quark distributions and the determination of the quark transversity distributions require semi-inclusive measurements, in which the detected final-state hadron reveals information about the spin, flavor, and charge of the "struck" quark participating in the deep-inelastic process. The $12 \mathrm{GeV}$ Upgrade will provide a unique opportunity to perform semi-inclusive measurements with high precision over a wide kinematic range, producing a detailed picture of the spin structure of the nucleon. Furthermore, semi-inclusive scattering allows us to probe the transverse momentum distribution of quarks in the nucleon via the azimuthal distribution of final-state hadrons. The study of these effects has made significant progress lately [Co93, Br02, Co02, Bu02a, Po03a] and is one of the frontiers of present research in QCD. Finally, the wide kinematic coverage of JLab at $12 \mathrm{GeV}$ provides a 


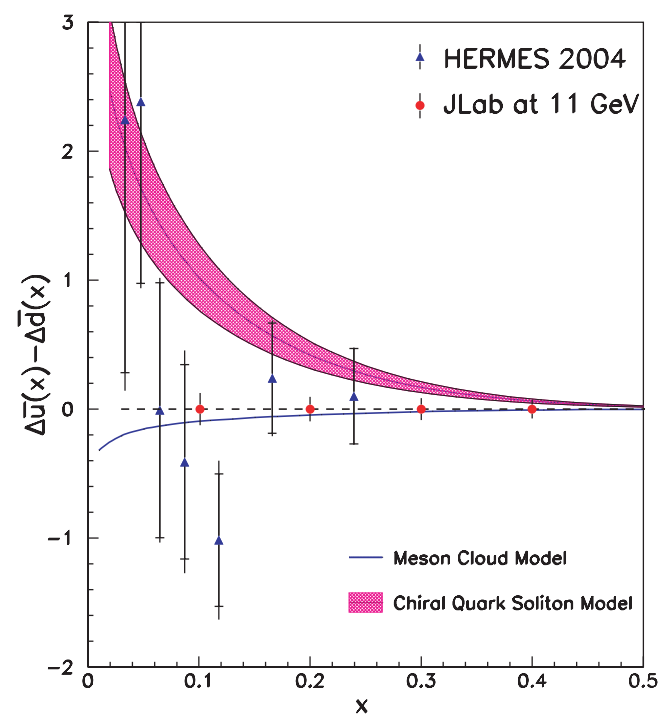

Figure 14: The polarized antiquark flavor asymmetry, $\Delta \bar{u}(x)-\Delta \bar{d}(x)$, as measured in semi-inclusive $e N$ scattering. The red dots show the projected accuracy of the measurements at $12 \mathrm{GeV}$. Existing data from HERMES (blue triangles) cannot discriminate between the different model predictions.

unique opportunity to improve our understanding of the hadronization process and gives access to the poorly understood target fragmentation region.

Flavor decomposition of quark spin. One of the most exciting discoveries of the past decade has been that the sea quarks are not flavor-symmetric. There is a significant excess of $\bar{d}$ antiquarks over $\bar{u}$ in the proton [Am91, Ba94, Ac98, Ha98]; see Ref. [Ga01] for a review. This large asymmetry cannot be explained by perturbative QCD (gluon bremsstrahlung) and highlights the crucial role played by non-perturbative physics in both the valence and sea quark structure of the proton. Theoretical explanations have focused on the role of dynamical chiral symmetry breaking and the associated pion cloud of the nucleon [Th83], as well as on the Pauli Exclusion Principle [Fi77, Sc91, Me99]. The best way to disentangle these effects is to measure the spin dependence of the antiquark flavor asymmetry. This can be done in semi-inclusive polarized $\vec{e} \vec{N}$ scattering at $12 \mathrm{GeV}$, see Fig. 14. The chiral quark-soliton model of the nucleon predicts a large asymmetry [Di96], in stark contrast to the "meson cloud" models, in which the $q \bar{q}$ pairs are strongly correlated into spin-zero pions and therefore do not contribute to the helicity distributions. Existing data from HERMES cannot discriminate between these scenarios. The expected data with the $12 \mathrm{GeV}$ Upgrade will provide a definitive answer. We note that these measurements of $\Delta \bar{u}(x)-\Delta \bar{d}(x)$ at $x>0.1$ will complement planned measurements of the flavor asymmetry at smaller $x$ via $W^{ \pm}$-boson production in high-energy $p p$ scattering at RHIC.

Quark transverse spin distributions and quark transverse momentum in semiinclusive scattering. The $12 \mathrm{GeV}$ Upgrade of CEBAF will provide additional, powerful new tools to understand the origin of the nucleons' spin. Measurements of the Collins azimuthal asymmetry in semi-inclusive pion production with a transversely polarized target will allow us to measure the quark transverse spin ("transversity") distributions. Figure 15 illustrates the qualitative improvement these data will provide relative to previous experiments (HERMES). Measurements of 

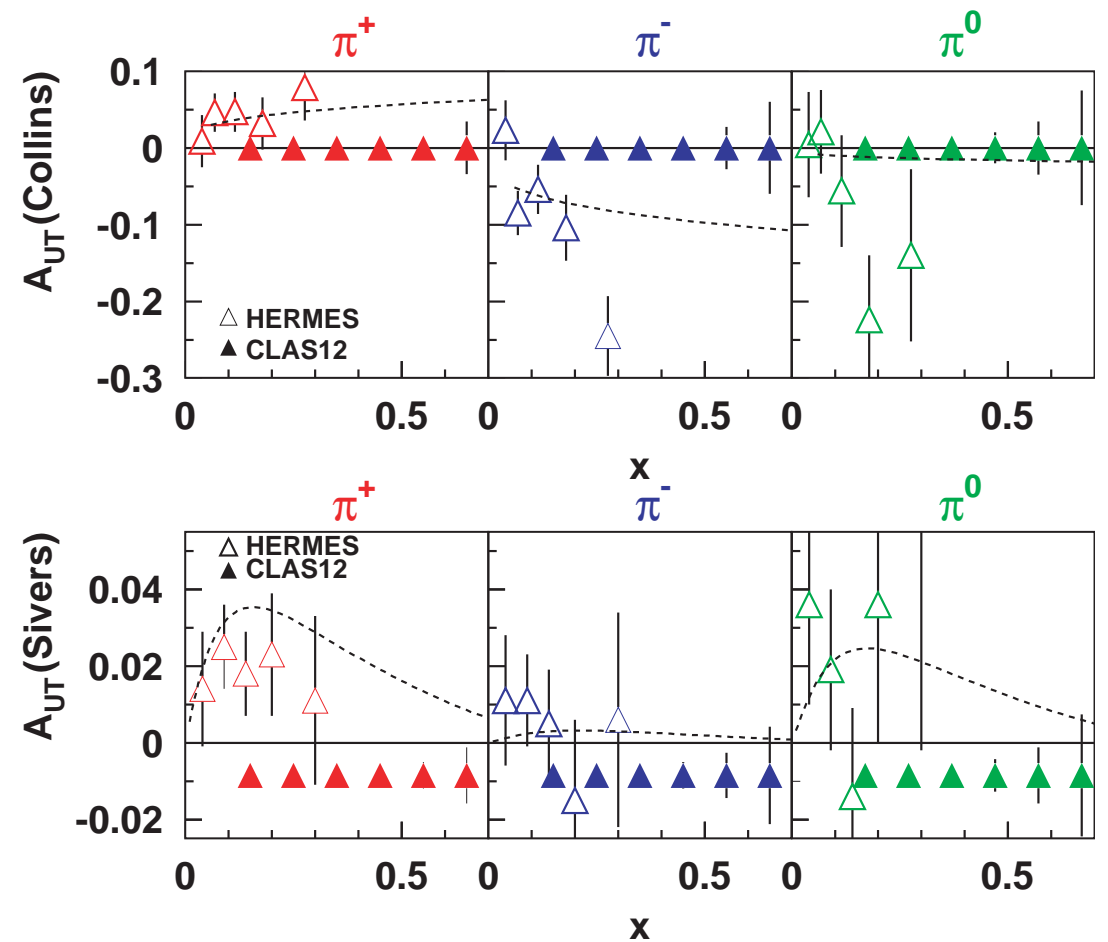

Figure 15: Projected measurements of the Collins and Sivers transverse single-spin asymmetries in semi-inclusive pion production at $12 \mathrm{GeV}$. The HERMES data are shown for comparison. The curves represent the phenomenological parameterizations of Ref. [Ef04].

the Sivers asymmetry (final state photons or hadrons in semi-inclusive deep-inelastic scattering) probe the transverse momentum distributions (TMD's) of quarks in the nucleon through its sensitivity to the interactions of the "struck" quark with the target remnants [Co93, Br02, Co02]. Figure 15 illustrates the qualitative improvement in the determination of these asymmetries compared to previous experiments. These data, together with precision data from inclusive spin structure function measurements at $12 \mathrm{GeV}$, will provide a comprehensive picture of the orbital motion of quarks and antiquarks in the nucleon for the first time.

The parton-hadron transition. A major unresolved issue in strong interaction physics is how the parton structure of the nucleon probed in scattering experiments with high momentum transfer, $Q^{2} \gg 1 \mathrm{GeV}^{2}$, turns into the hadronic structure observed at low momentum transfer, $Q^{2} \ll 1 \mathrm{GeV}^{2}$. Exploring this transition is crucial for understanding the non-perturbative effects determining hadron structure in QCD - confinement and the spontaneous breaking of chiral symmetry. On the practical side, a quantitative understanding of the limits of the perturbative QCD description is a prerequisite for experiments aimed at mapping the quark and gluon structure of the nucleon. The $12 \mathrm{GeV}$ Upgrade would allow for comprehensive studies of the transition from the "partonic" to the "hadronic" regime. It provides the necessary luminosity and $Q^{2}$-range to perform accurate measurements of the structure functions in the resonance region over a wide range of $Q^{2}$. The excellent kinematic coverage in the Bjorken variable, $x$ (see Fig. 3), and the design of 


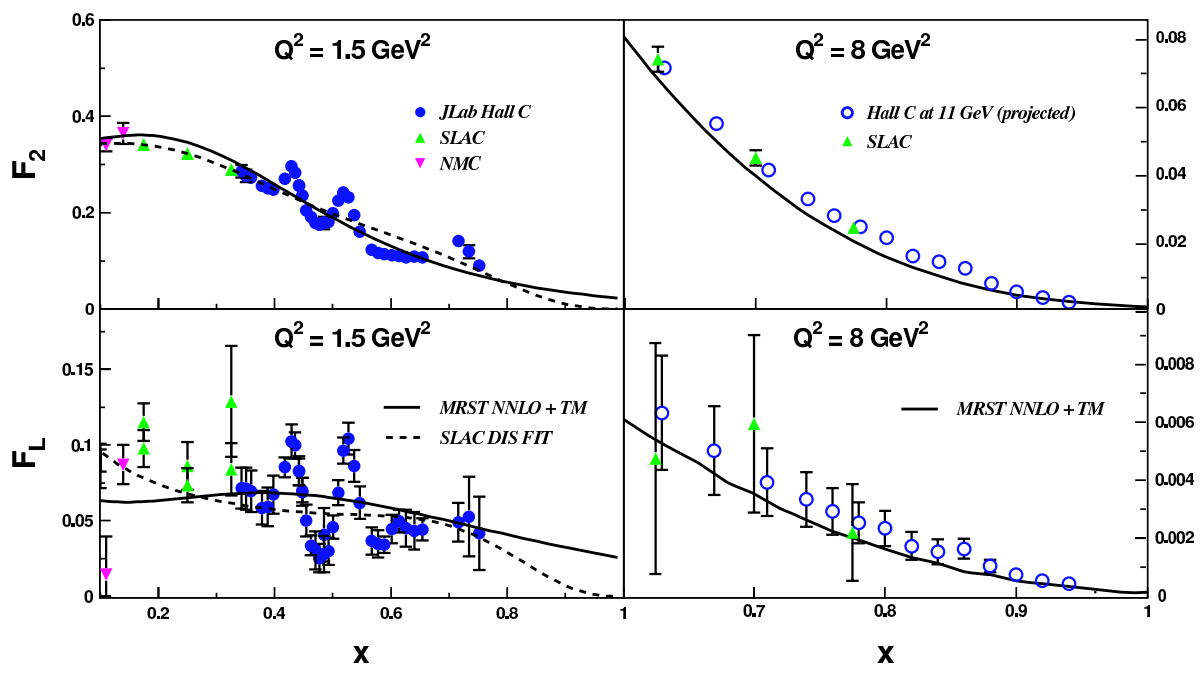

Figure 16: The potential of the $12 \mathrm{GeV}$ Upgrade for exploring quark-hadron duality in the nucleon structure functions $F_{2}$ (upper row) and $F_{L}$ (lower row). The left panels show the Hall $\mathrm{C}$ data taken at $6 \mathrm{GeV}$, the right panels the projected high- $x$ and high- $Q^{2}$ data at $12 \mathrm{GeV}$.

the detectors make it possible to measure moments of structure functions (integrals over energy), which exhibit a particularly simple relation to hadronic observables at low $Q^{2}$ (sum rules).

Quark-hadron duality. Deep-inelastic eN scattering at moderate energies (CM energy $W \sim 1-2 \mathrm{GeV}$ ) allows us to study the transition from the coherent excitation of individual nucleon resonances to the incoherent scattering off quarks with increasing energy, and its change with the momentum transfer, $Q^{2}$. Existing experimental data show a striking similarity between the structure functions measured in the resonance region and the high $-Q^{2}$ partonic parameterizations when extrapolated to low $Q^{2}$ ("quark-hadron duality"), see Fig. 16. This phenomenon shows that the parton structure of the nucleon observed at high $Q^{2}$ is encoded in certain gross features of its excitation spectrum. Recently, significant progress has been made in quantifying and understanding quark-hadron duality; see Ref. [Me05] for a review. The $12 \mathrm{GeV}$ Upgrade will allow us to extend these studies to a much wider range of $Q^{2}$ and $x$. In particular, to understand the dynamical origins of duality it is necessary to separate the cross sections for longitudinal and transverse photon polarization (L/T separation) and to unravel the flavor structure by measurements with proton and neutron (i.e., nuclear) targets. In channels where duality is demonstrably valid, the quark distribution functions can be reconstructed at higher $x$ than it is possible in direct measurements at high $Q^{2}$. Finally, we plan to extend the study of duality to semi-inclusive hadron production, where they serve to explore the region of validity of the QCD fragmentation mechanism.

Structure function moments and the Gerasimov-Drell-Hearn integral. The large kinematic range provided by the $12 \mathrm{GeV}$ Upgrade will also allow us to determine moments $(x-$ weighted integrals) of both polarized and unpolarized structure functions with unprecedented accuracy, and follow their $Q^{2}$-dependence. Moments of the polarized structure functions $g_{1}$ and $g_{2}$ contain information about the color-electric and magnetic polarizabilities of the nucleon, which characterize the response of the gluon fields to the nucleon's polarization. These moments are also calculable from first principles using lattice QCD simulations [Do02, De02], and will thus provide 
critical tests of QCD itself.

Of particular interest is the Gerasimov-Drell-Hearn (GDH) integral of the spin structure function, whose value at $Q^{2}=0$ is fixed by the GDH sum rule for the real photoabsorption cross section. Existing data show a spectacular mismatch between the high- $Q^{2}$ values and the $Q^{2}=0$ value determined from photoproduction experiments. The GDH integral thus offers an excellent testing ground for dynamical models of the transition from the partonic to the hadronic regime. Its experimental determination requires measurements over a wide range of energies, corresponding to the resonance region as well as the deep inelastic scattering regime. At $6 \mathrm{GeV}$, measurements are confined largely to the resonance region. With the $12 \mathrm{GeV}$ Upgrade, we shall have the kinematic coverage necessary to probe the high-energy piece of the extended GDH integral, and map its $Q^{2}$-evolution. This program will bring GDH studies to a new level of quantitative precision.

\section{C The Physics of Nuclei}

Lying at the core of every atom, at the $10^{-15} \mathrm{~m}$ scale, the nucleus comprises over $99 \%$ of the atom's mass. It is a unique many-body system that can be understood quantitatively as assemblies of individual protons and neutrons bound by an effective nuclear force. Nuclear physics describes the dominant two-body $N N$ force at "long range" (the radius of the nucleus or more precisely the pion Compton wave length) as being mediated by the exchange of pions, the lightest hadron Nature provides. This attractive force is delicately balanced by a force at shorter distances that repels. When protons and neutrons are at "short range", the nuclear force reverses and they repel each other. This delicate interplay is not well understood, yet it enables the existence of atomic nuclei and the chemical elements.

At $12 \mathrm{GeV}$, we can probe the interesting details of the force at distance scales much less than the pion Compton wave length, where the effects of two-pion exchange, vector meson exchange, and quark exchange all compete, and where the contributions from virtual baryon resonances (such as the $\Delta$ ) might be resolved. Although well constrained phenomenologically by the large body of $p p$ and $n p$ elastic scattering data, it is not yet understood under what circumstances the effective nuclear force can be described in terms of the exchange of mesons, and when it is more efficient to describe the force in terms of the underlying quark-gluon exchange forces.

If the nuclear force is the residue of the even stronger QCD force between quarks, forever confined in the nucleons, are protons and neutrons under every circumstance the best quasi-particles to describe the nucleus, or can we still see an imprint of the basic quarks and gluons? After many decades of research, the basic mystery of the origin of nuclei still remains. How did nuclei emerge from QCD? This is one of the key issues of modern-day studies of nuclei [Gu04a].

Alternatively, Nature provided in the atomic nucleus, with a size comparable to the range of the QCD and nuclear forces, a perfect laboratory to study how the underlying QCD non-Abelian degrees of freedom, such as its elusive color charge, manifest themselves. The idea is here to strike a quark inside the nucleus with such velocity that one can uniquely witness how hadrons emerge, as they must, from these speedy quarks (and the associated gluons) on their path through the nuclear theater. Our present sketchy understanding of this process will be vastly improved with the $12-\mathrm{GeV}$ program at JLab. 


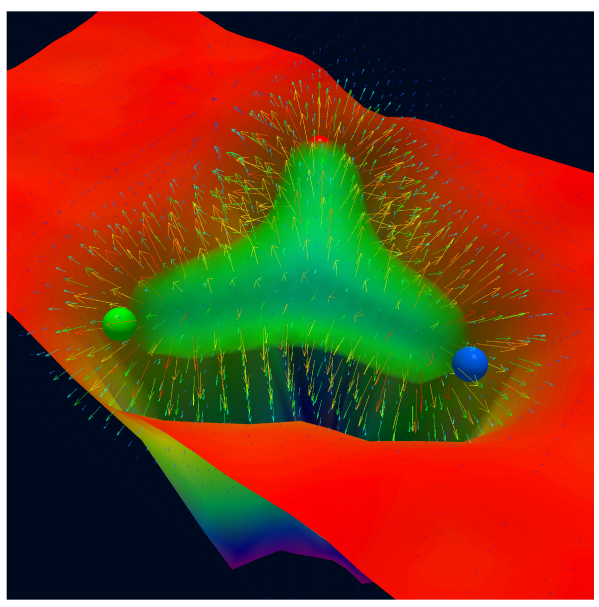

Figure 17: A visualization based on lattice QCD calculations. A baryon is shown (in the static approximation) in the presence of the QCD vacuum. The vacuum action density in a plane passing through the centers of the quarks is represented by the red surface; this vacuum is visibly 'expelled' by the presence of the baryon. This demonstrates the principle that nucleons in a nucleus experience a different, diminished QCD vacuum relative to free protons and neutrons, an effect which alters their structure within the nuclear medium.

The Emergence of Nuclei from QCD. Studies of scattering between two nucleons at low energy demonstrate that their interactions can be described in part in terms of the exchange of mesons. This insight is the basis for many successful models of nuclear structure. However, the fundamental constituents of nuclei are quarks and gluons, whose interactions are described by QCD. Both nucleons and mesons are composites of quarks, that can not exist in isolation due to confinement. This leads to some of the most fundamental questions in modern nuclear physics:

- How do the nucleon-based models of nuclear physics with interacting nucleons and mesons arise as an approximation to the quark-gluon picture of QCD?

- In probing ever-shorter distances within the nucleus, what is the role of the fundamental constituents of QCD - quarks and gluons - in the description of nuclei?

- Does the nuclear environment modify the quark-gluon structure of nucleons and mesons?

The partonic structure of nuclei. Nucleons and mesons are the building blocks of ordinary nuclear matter, but there is no guarantee that these building blocks have properties in nuclei identical to those of the isolated hadrons. The neutron life time in nuclei is definitely changed! In QCD, the properties of hadrons are strongly influenced by the induced sea of quark-antiquark pairs and the gluons produced in the confining interactions. Recent Lattice QCD calculations indeed verify that the probability of finding virtual quark-antiquark pairs in the QCD vacuum decreases systematically when quarks are added. Hence, a hole or depletion in the QCD vacuum is created, a picture qualitatively similar to the bag model. This is graphically illustrated in Fig. 17.

The question of whether a nucleon bound in the nuclear medium has different properties from those of a free nucleon has been a long-standing issue in nuclear physics. It was first considered seriously with the discovery of the nuclear EMC effect some twenty years ago, in which scattering 


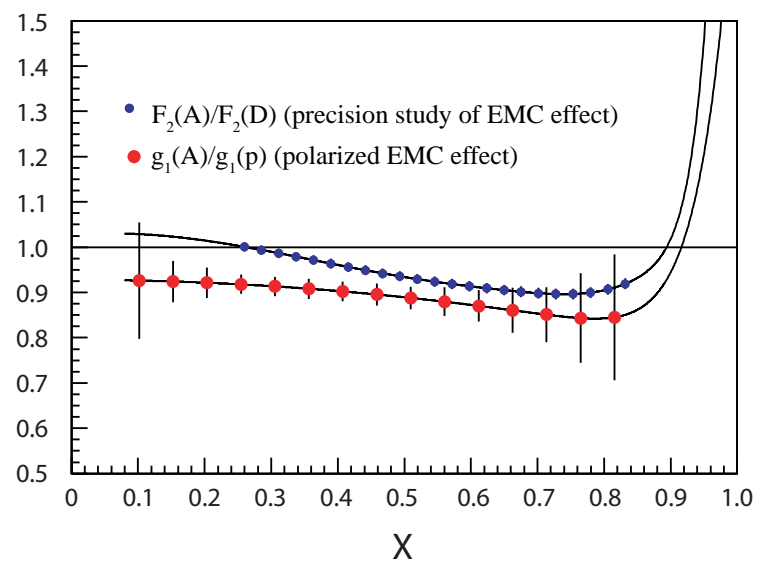

Figure 18: An illustration of the polarized and unpolarized EMC effect studies accessible following the upgrade. The unpolarized measurement shows representative uncertainties for precision studies performed on a variety of nuclei. The polarized measurement compares $g_{1}$ in ${ }^{7} \mathrm{Li}$ and in ${ }^{1} \mathrm{H}$.

from quarks inside the nucleus was discovered to differ in non-trivial ways from the scattering of quarks in a free nucleon. Existing measurements indicate little $Q^{2}$ dependence, and an $A$ dependence in the magnitude, but not the overall form, of the structure function modification in nuclei. The nature of the modifications in nuclei depends primarily on Bjorken- $x$; its most prominent features are an enhancement in the region $0.1<x<0.3$ and a depletion in the region $0.3<x<0.7$. Despite a huge world-wide effort in experiment and theory, the EMC effect remains a mystery. Explanations of it are hampered by the lack of a theory that can consistently account for the nuclear dependence of the quark distributions over a large range of $x$. Further difficulties arise from the lack of evidence for the surplus of antiquarks predicted to arise from pions being exchanged between nucleons (as was observed in Drell-Yan processes). The failure to account for these hard scattering results is a serious problem for nuclear theory.

Knowing that the valence quark region is depleted does not tell us enough to understand the origin of the EMC effect. We would like to know if $u$ and $d$ valence quarks are depleted in the same manner. Although limits on the nuclear modification of the sea quarks exist from Drell-Yan data, some small modification must exist, and possibilities exist to map out the flavor dependence, like in semi-inclusive DIS studies discussed earlier. Even more straightforward, there is no experimental information on the spin dependence of the EMC effect, predicted to be twice as large as the regular EMC effect. A measurement of the spin-dependence of the EMC effect is well within the reach of the $12 \mathrm{GeV}$ upgrade. This spin-dependent EMC effect emphasizes the quark polarization degrees of freedom within a nucleus, due to the spin-dependence of the coupling between the quarks and the strong fields inside the nucleus. In Fig. 18 we highlight a possible measurement of the nuclear ratio of spin structure functions, $g_{1}$, in ${ }^{7} \mathrm{Li}$ and ${ }^{1} \mathrm{H}$.

The CW character of the CEBAF beam also opens a large window on measurements of structure functions in coincidence with low-energy nucleons or target fragments. This technique is currently being pioneered at JLab, with a recoil proton detector "tagging" scattering events on a nearly on-shell neutron in a deuteron target. The technique can easily be expanded to EMC-type measurements on ${ }^{2} \mathrm{H}$ and ${ }^{3} \mathrm{He}$. A glimpse at the information obtainable from such experiments has been provided by the analysis of deep-inelastic neutrino scattering events in heavy-liquid bubble chambers. In spite of the poor statistics, these experiments have shown that the structure func- 
tions tagged on low-energy protons are different from those determined from inclusive scattering. A large acceptance device at a $12-\mathrm{GeV}$ JLab will essentially function as a "bubble chamber" for deep-inelastic electron scattering events, and will allow reconstruction of the EMC effect from a complete measurement of the $x$-dependence of exclusive channels on light nuclei.

Short-range structure of nuclei. The short range repulsive core is a critical component of the $N N$ force, and leads to the saturation necessary for the formation of stable nuclei. It affects about one third of the nucleons in a nuclei, resulting in shell occupations well above the Fermi sea that substantially exceed the predictions of nuclear shell models. While the existence of this extremely strong repulsive core has been a feature of $N-N$ potentials for many years, its QCD origin remains unclear. Two nucleons at close enough proximity (within about $1 \mathrm{fm}$ ) will consequentially be boosted to high momentum. Such high momentum components remain one of the most poorly understood aspects of nuclear structure.

Alternately, these high momentum nucleons can be used to experimentally point to high density "droplets" of nuclear matter. Configurations where two nucleons are, as above, separated by less than $1 \mathrm{fm}$, correspond to regions of overlap with local densities several times larger than average nuclear densities. Such densities can approach or even exceed the densities found in neutron stars, supernovae, or other compact astronomical objects. The structure of nucleons and the distribution of high-momentum quarks may be substantially altered in this region, where significant overlap of nucleons should allow direct interaction between quarks in different nucleons. This may yield a dramatic increase in the probability of finding "superfast" quarks - quarks that have gained more momentum than they could have had in an isolated nucleon (see Fig. 19). The individual identity of the nucleons may even be lost, and an ephemeral state may exist that is better represented as a single six-quark object, rather than a pair of independent, strongly interacting nucleons. In fact, the observation of the nuclear EMC effect may already hint that quark confinement is weakened at densities found inside a nucleus. For nucleons at short distance, the impact of the altered QCD vacuum as illustrated in Fig. 17 will increase. Hence, probing these high-density configurations may allow us to learn about the transition from the conventional picture of nuclear physics to more exotic states of QCD at high density, also complementing the high-temperature studies at RHIC and LHC.

The onset of scaling behavior in nuclear reactions. QCD becomes scale invariant in the limit of weak coupling. The primary empirical evidence for scale-invariance in hadron physics at short distances is the Bjorken scaling of the deep inelastic cross section electron-proton at momentum transfers beyond $Q^{2} \geq 1 \mathrm{GeV}^{2}$. Other manifestations of the underlying scale-invariance of hadron physics are dimensional counting rules for hard exclusive processes: $\frac{d \sigma}{d t} \sim 1 / s^{n-2}$, where $n$ counts the minimum number of elementary constituents of a hadron or elementary field. In the limit of zero coupling, the scale-invariance of nuclear reactions directly corresponds to the conformal symmetry of the underlying quantum field theory. A major surprise remains the finding of apparent scale invariance of nuclear reactions in kinematic regions where the coupling is not small yet.

Scaling in the differential cross section $d \sigma / d t$ has been pursued experimentally for many years as providing signatures of the transition from the meson/nucleon description of nuclei to the underlying quark and gluon description. The deuteron photodisintegration reaction, $\gamma d \rightarrow p n$, is one of the simplest reactions for studying explicit quark effects in nuclei. In recent years, extensive studies of 


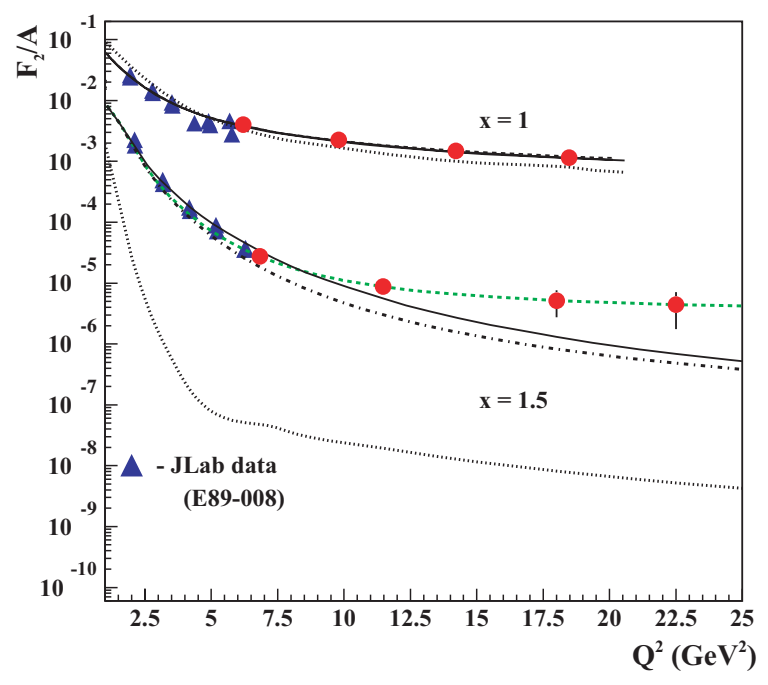

Figure 19: Prediction for the onset of Bjorken scaling for the ${ }^{56} \mathrm{Fe}\left(e, e^{\prime}\right) X$ reaction. The solid line and the dash-dotted line are predictions with two-nucleon correlations, without and with the EMC modification of the nucleon elastic form factor, respectively. The dashed line is the prediction with multinucleon correlations with EMC effects included, and the dotted line is the prediction of mean-field approximation. The actual value of the $F_{2}$ structure function in the scaling region, at $x$ $=1.5$, may be dramatically changed due to small six-quark contributions.

deuteron photo-disintegration have been carried out at SLAC and JLab [Na88, Sc01]. Figure 20 shows the scaled differential cross-section $\left(s^{11} d \sigma / d t\right)$ for deuteron photodisintegration as a function of photon energy. The available data [Sc01] seem to show scaling at $70^{\circ}$ and $90^{\circ}$, and suggest the onset of scaling at higher photon energies at $53^{\circ}$ and $37^{\circ}$. The threshold for this scaling behavior corresponds to a transverse momentum slightly over $1 \mathrm{GeV}$. Theoretical efforts [Fr01, Ko93, Gr01, Rapc] to describe this behavior agree qualitatively with the data, but do not reproduce them precisely. While none of the theories agree with all of the data as well as one would like, they do indicate that quark models can approximately reproduce the cross section data, and therefore confirm the importance of the deuteron photodisintegration process in the study of the transition region. The Upgrade will permit the extension of these data to photon energies near $8 \mathrm{GeV}$, as shown in the figure, permitting a validation of the apparent constant transverse momentum onset of scaling behavior.

While global scaling behavior has been observed in many exclusive processes [An76], no experimental evidence supports hadron helicity conservation, which was predicted in the same approach, in the similar energy and momentum transfer region. One would anticipate an earlier onset of scaling or hadron helicity conservation in ratios of cross sections, where non-perturbative effects may cancel. The simplest of such ratios is the charged pion photoproduction differential cross-section ratio, $\frac{d \sigma}{d t}\left(\gamma n \rightarrow \pi^{-} p\right) / \frac{d \sigma}{d t}\left(\gamma p \rightarrow \pi^{+} n\right)$, as nucleon photopion production processes decrease relatively slowly with energy compared to other photon-induced processes (quark counting rules predict a $s^{-7}$-dependence for the differential cross-section). Calculations of this ratio have been performed in the framework of the handbag mechanism [Hu00, Hu03], in which the amplitude is factorized into a parton-level subprocess $\gamma q_{a} \rightarrow P q_{b}$ and GPDs. The GPD part of the contribution describing the soft hadron-parton transitions indeed cancels in this ratio provided the assumption of negligi- 


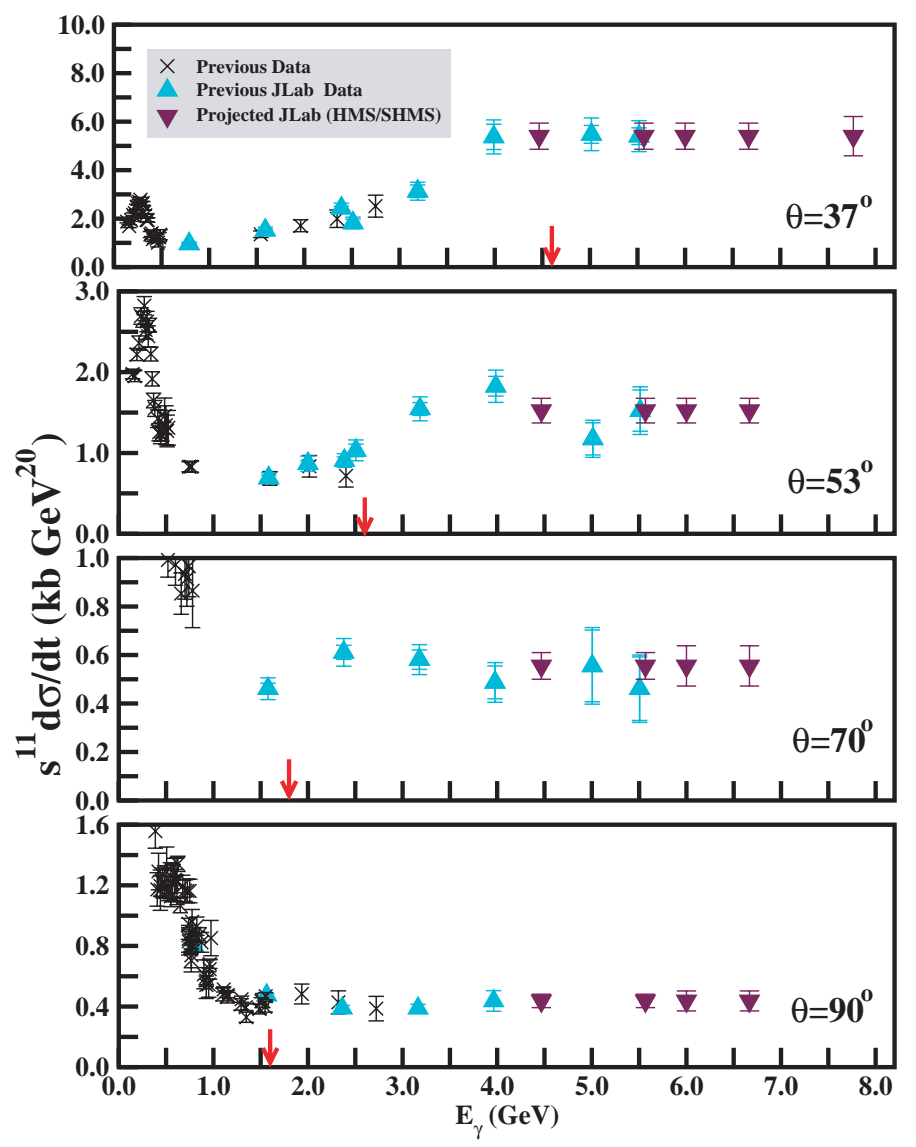

Figure 20: Available data and projected results for a differential cross-section measurement of deuteron photodisintegration. The arrow indicates a transverse momentum of $1.6 \mathrm{GeV}$ in each plot. 


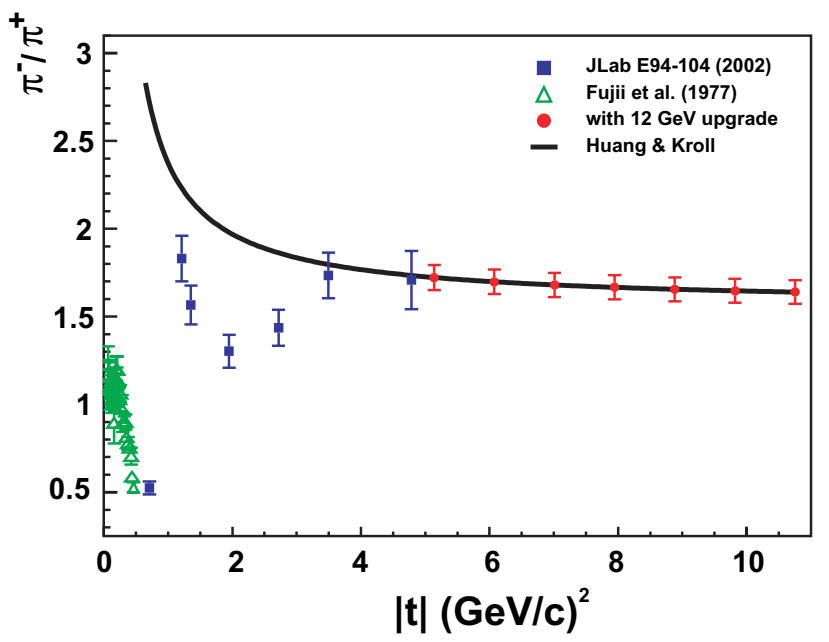

Figure 21: The charged pion photoproduction differential cross-section ratio at a C.M. angle of $90^{\circ}$, as a function of $|t|(\mathrm{GeV} / \mathrm{c})^{2}$ (blue) along with the projected measurements for JLab at $11 \mathrm{GeV}$ (red). The solid curve is a prediction by Huang and Kroll [Hu00].

ble quark helicity flip contributions and the dominance of a helicity conserving amplitude of the parton-level subprocess $\gamma q_{a} \rightarrow P q_{b}$ for pseudoscalar meson production [Hu03]. The most recent charged pion ratio data [Zh03] from experiment E94-104 for momentum transfers up to $5.0 \mathrm{GeV}^{2}$ indicate that indeed one of the helicity conserving amplitudes dominates. This ratio measurement can be extended to a $|t|$ value of about $10 \mathrm{GeV}^{2}$ with an $11 \mathrm{GeV}$ beam. Figure 21 shows both the available data and projected results for this ratio at $11 \mathrm{GeV}$, together with a prediction of Huang and Kroll [Hu00]. In addition, the assumption of negligible helicity-flip amplitudes can be tested by polarization measurements such as the $\vec{\gamma} p \rightarrow \vec{p} \pi^{0}$ reaction. The upgrade will permit a doubling of the present energy range of this experiment to $8 \mathrm{GeV}$.

Fundamental QCD processes in the nuclear arena. The $12 \mathrm{GeV}$ Upgrade will provide unique opportunities for an understanding of how hadron-hadron interactions emerge from the underlying quark-gluon structure of QCD. The attribute that sets QCD apart from other successful field theories is that it is non-Abelian. This gives the gauge bosons - the gluons - color charge and causes them to strongly interact with each other. Ultimately, this is the origin of confinement of quarks in hadrons. Here, we will use this confinement property as a tool: quarks, when struck vehemently in an $11 \mathrm{GeV}$ scattering process, must turn into hadrons over a range comparable to the size of the nucleus. This will allow, for example, studies of quark propagation and hadronization in the nuclear medium, the onset of the phenomenon of color transparency in exclusive processes, and color van der Waals-type interactions in $J / \psi$-meson photoproduction.

Quark propagation through cold QCD matter: nuclear hadronization and transverse momentum broadening. The properties of isolated quarks are generally experimentally inaccessible due to quark confinement in hadrons. In hard interactions, such as in deep inelastic scattering, the struck quark in a nucleon must separate from the rest of the residual system. When this separation distance is comparable to nuclear radii, it is possible to study the properties of the propagating quark by varying the radii of the nuclear targets and observing modifications of 


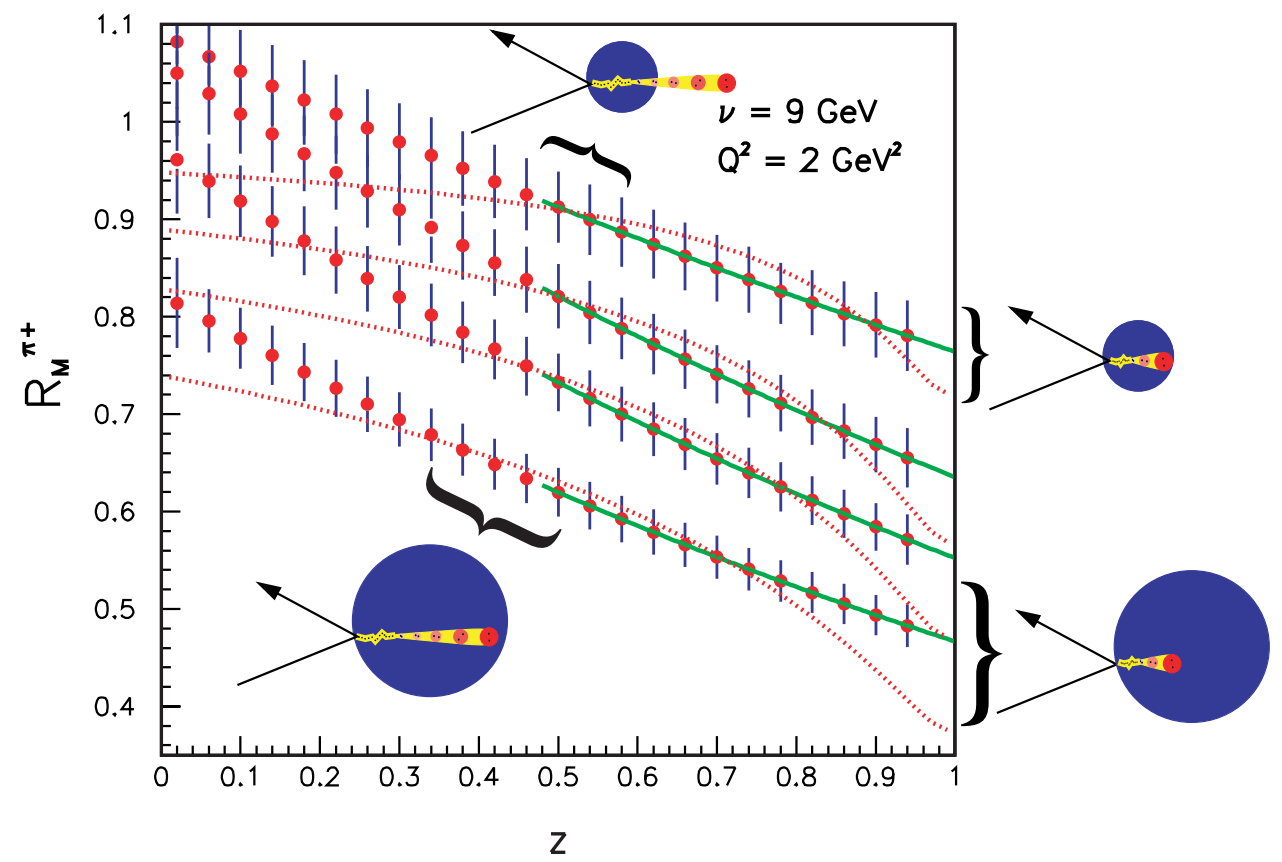

Figure 22: Hadron attenuation in nuclear deep inelastic scattering, shown by the pion multiplicity ratio vs. $z$ for fixed $Q^{2}$ and $\nu$. The cartoons indicate the regions of the data where the pion formation length ranges from longer to shorter in larger and smaller nuclei, schematically demonstrating how the formation lengths can be measured experimentally. The curves from top to bottom correspond to nitrogen, argon, krypton, and gold; the green line is a gluon bremsstrahlung model calculation, and the red dotted line is a parameterization derived from HERMES data. The figure is an example of the large amount of information (for a number of different hadrons) that will be accessible with the $11 \mathrm{GeV}$ beam.

the final hadronic states (see Fig. 22). Presently, the formation of final hadrons due to confinement is explained heuristically by sketches of space-time processes involving string breaking. With $11 \mathrm{GeV}$ electrons, we can map the distances over which the energetic colored quark transforms into a colorless hadron, and characterize this hadronization as a function of multiple variables. This provides completely new information on how the color field of the hadron is restored in real time through the fundamental process of gluon emission. The analogous process has been studied and understood in QED. The propagating quark is expected to experience some interaction with the nuclear medium. One prediction is that it undergoes multiple soft scatterings mediated by gluon emission. In this picture, the quark experiences a medium-induced energy loss that may be experimentally accessible and which may exhibit exotic coherence phenomena. This process measurably broadens the transverse momentum distribution of the hadron emerging from larger nuclei. It is anticipated that a quark-gluon correlation function, and the quark energy loss, can be extracted from the measured broadening. The topics of color field restoration by gluon emission, quark-gluon correlations, and quark energy loss, offer fundamental and interesting insights into the nature of QCD and confinement. In addition, they are of very high interest in the study of relativistic A-A and p-A collisions, where they are basic and essential ingredients that must be understood. 
Color transparency. The nature of hadronic interactions can be investigated via tests of the prediction of "color transparency". Color transparency is one of the few direct manifestations of the underlying color degrees-of-freedom in nuclear physics. Under the right conditions, three quarks, each of which (alone) would have interacted very strongly with nuclear matter, could form an object that passes undisturbed through the nuclear medium. A similar phenomenon occurs in QED, where an $e^{+} e^{-}$pair of small size has a small cross section determined by its electric dipole moment. In $\mathrm{QCD}$, a $q \bar{q}$ or $q q q$ system with a small color dipole moment is predicted to have similarly reduced interactions due to cancelation of the color fields of the quarks. While the $q \bar{q}$ case is completely analogous to the QED example, color transparency in the $q q q$ case would be one of the rare demonstrations of the $S U(3)$ nature of the underlying color degrees of freedom. While the nucleonic example is more exotic, meson production may provide a more practical setting for observing this phenomenon. Intuitively, one expects an earlier onset of color transparency for meson production, as it is much more probable to produce a small sized configuration in a $q \bar{q}$ system than in the $q q q$ system. Color transparency can be observed experimentally by measuring a reduced attenuation of particles as they exit a nucleus, or by measuring a decrease in production of particles produced via two-step rescattering mechanisms, which allows the use of few-body nuclei. A series of attenuation and rescattering measurements for both protons and mesons will allow us to separate the necessary ingredients: formation of the small sized configuration, the reduced color interaction of these configurations, and the evolution of these exotic configurations back into ordinary hadrons.

\section{D Symmetry Tests in Nuclear Physics}

Precision parity-violating electron scattering experiments made feasible by the $12 \mathrm{GeV}$ Upgrade have the sensitivity to search for deviations from the Standard Model that could signal the presence of new gauge bosons $\left(Z^{\prime} \mathrm{s}\right)$, the existence of leptoquarks, or particles predicted by supersymmetric theories, i.e. physics beyond the Standard Model. Planned studies of the three neutral pseudoscalar mesons, the $\pi^{0}, \eta$ and $\eta^{\prime}$, will provide fundamental information about low energy QCD, including certain critical low energy parameters, the effects of $\mathrm{SU}(3)$ and isospin breaking by the $u, d$, and $s$ quark masses, and the strengths of the two types of chiral anomalies. These two programs are described briefly below.

Standard model tests. Parity-violating electron scattering (PVES) is one of the most powerful techniques to measure low energy weak neutral current (WNC) processes. Precision measurements of WNC scattering processes, mediated by the $Z^{0}$ boson, are potentially sensitive to new physics amplitudes beyond the Standard Model of electro-weak interactions. Such indirect searches via precision measurements are complementary to potential direct discoveries of new particles with $\mathrm{TeV}$ scale masses at high energy colliders. For example, WNC measurements can provide constraints on the couplings of such new particles to leptons and quarks. In addition to searching for new physics, PVES with judicious choice of kinematics and target nucleus can provide information on novel aspects of hadron structure. The $12 \mathrm{GeV}$ upgrade will greatly extend the ongoing JLab program along these two exciting avenues of discovery.

One of the more compelling new opportunities will be a remarkably accurate measurement of the weak charge of the electron, via the parity-violating asymmetry in electron-electron (Møller) scattering. Such a measurement becomes feasible with the upgraded JLab electron beam, which constitutes a unique combination of high energy and unprecedented luminosity and stability. The 


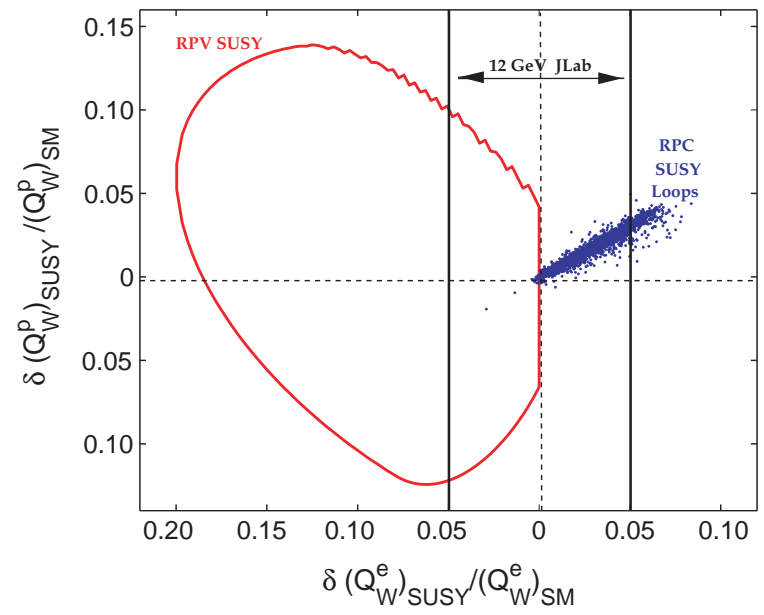

Figure 23: Relative shifts in $Q_{\text {weak }}^{e}$ and $Q_{\text {weak }}^{p}$ from SUSY effects (at 95\% confidence level unless otherwise specified). The blue dots denote MSSM loop corrections for approximately 3000 SUSYbreaking parameter choices. The interior of the red, truncated elliptical contour shows allowed shifts due to R-parity violation. Because the lightest supersymmetric particle is unstable if Rparity is not conserved, these SUSY models offer no candidate for dark matter [Ku02]. The vertical bars illustrate what could be achieved by a $12 \mathrm{GeV}$ Møller experiment at Jefferson Laboratory.

small size of the predicted asymmetry, 40 parts per billion, will require extraordinary control of systematic errors, making it appropriate as a next generation measurement. The completion of the currently approved program of PVES measurements will ensure the development and testing of the necessary instrumentation for the measurement.

The weak charge of the electron is suppressed in the Standard Model by the factor 1 $4 \sin ^{2} \theta_{W} \simeq 0.05$, where $\theta_{W}$ is the weak mixing angle. Because new processes beyond the Standard Model will not generally be suppressed by the same factor, this measurement is a powerful window into such processes. The achievable accuracy of the electron weak charge measurement provides extraordinary sensitivity to electron substructure ("compositeness") to a scale of nearly $30 \mathrm{TeV}$, corresponding to 7 millionths of a Fermi. Such sensitivity to leptonic substructure will be unmatched anywhere in the world until the advent of a linear collider or a neutrino factory.

The measurement is also sensitive to the existence of new neutral gauge bosons in the range of 1 to $2 \mathrm{TeV}$; such model-dependent limits are comparable to those to be achieved by measurements at the Large Hadron Collider. Furthermore, the measurement will severely constrain the viability of SUSY models which violate R-parity. This has implications for a plausible SUSY dark matter candidate to explain some of the non-luminous and unexplained source of $90 \%$ of the mass of the universe. (See Fig. 23.) Even in the context of the Standard Model, an improved measurement will have significant consequences. As a measurement of the weak mixing angle, it will be the most precise low energy determination. Indeed, the precision will be on par with the current best high energy measurements. This may shed light on the significant discrepancy at the Z-pole between purely leptonic and semi-leptonic measurements of the weak mixing angle.

The upgraded beam energy will also make possible measurements of parity violation in deep inelastic scattering (PVDIS). On an isoscalar target at moderate $x$ PVDIS is also sensitive to $\sin ^{2} \theta_{W}$. In fact, it was a measurement of this process by the SLAC E122 experiment [Pr78] that 
established the Standard Model as the theory of the neutral weak interaction. One interpretation of a recent measurement of neutrino-nucleus DIS by the NuTeV collaboration is that the Standard Model is incomplete. A measurement of the E122 PVDIS asymmetry, with an order of magnitude improvement in accuracy, will test the electro-weak theory with comparable sensitivity. This measurement becomes feasible with a $11 \mathrm{GeV}$ beam and the presently planned detectors in one of the high luminosity end stations, with rather modest demands on beam time and systematic control.

Such tests of the electro-weak theory in DIS require a thorough understanding of possible nucleon structure effects that could cloud the interpretation of the measurements. These effects are important to study in themselves and have the potential to provide profound new insights into the dynamics of quarks inside nucleons. Examples of potential PVDIS measurements are the value of $d(x) / u(x)$ as $x \rightarrow 1$, the search for evidence of charge symmetry violation (CSV) at the partonic level, and the characterization of novel higher twist effects. These topics can be investigated with moderate beam time using a new large-acceptance spectrometer/detector package and an upgraded JLab beam. Thus, quite apart from providing a sound basis for the interpretation of a PVDIS measurement to search for physics beyond the Standard Model, this program can lead to important new discoveries regarding the quark structure of nucleons.

Recently, two workshops were held at Jefferson Laboratory to discuss the physics potential and the experimental feasibility of the Møller scattering and PVDIS programs with $11 \mathrm{GeV}$ beams, bringing together leading theorists and experimentalists in the field. In the case of Møller scattering, there was general consensus that a measurement of the parity-violating asymmetry with a total error smaller than 1 part per billion was feasible. There was overwhelming enthusiasm to aggressively proceed with the design of such a measurement that would yield a measurement of the weak mixing angle $\sin ^{2} \theta_{W}$ with an error between 0.0002 and 0.0003 . In the case of PVDIS, it was recognized that $1 \%$ asymmetry measurements at $x \sim 0.7$ would be attainable with a large volume, high field solenoidal spectrometer. Such measurements would have tremendous discovery potential. Apart from yielding complementary tests of the electroweak theory in the semi-leptonic sector, the potential observation of the onset of CSV and the characterization of higher twist effects might provide the cleanest way to go beyond the conventional quark-parton description of the nucleon.

Tests of Chiral symmetry and anomalies via the Primakoff effect. Two basic phenomena in QCD, namely the spontaneous breaking of chiral symmetry and the chiral anomalies, are manifested in their most unambiguous form in the sector of light pseudoscalar mesons. The chiral anomalies induced by the electromagnetic interactions drive the decays that are the centerpiece of this proposal, namely the two-photon decays of the $\pi^{0}, \eta$, and $\eta^{\prime}$. Another anomaly, the axial anomaly induced by QCD itself and which explicitly breaks the axial $U(1)$ symmetry, gives a large fraction of the $\eta^{\prime}$ mass. In addition, the explicit chiral symmetry breaking by the light-quark masses manifests itself in their decays.

The advent of $12 \mathrm{GeV}$ electron beams at Jefferson Laboratory will make it possible to extend recently completed high precision measurement of the $\pi^{0} \rightarrow \gamma \gamma$ decay width via the Primakoff effect to include the $\eta$ and $\eta^{\prime}$ mesons, and will also make possible a low $Q^{2}$ measurement of the transition form factors of these mesons. All these measurements will have a significant impact on the current knowledge of several fundamental aspects of QCD. Of particular importance is a more accurate determination of the quark-mass ratio $\left(m_{u}-m_{d}\right) / m_{s}$. Figure 24 illustrates the current situation where the closely related ratio $\mathcal{Q}^{2}=\left(m_{s}^{2}-\hat{m}^{2}\right) /\left(m_{d}^{2}-m_{u}^{2}\right)$ (where $\left.\hat{m}=\left(m_{u}+m_{d}\right) / 2\right)$ is obtained 


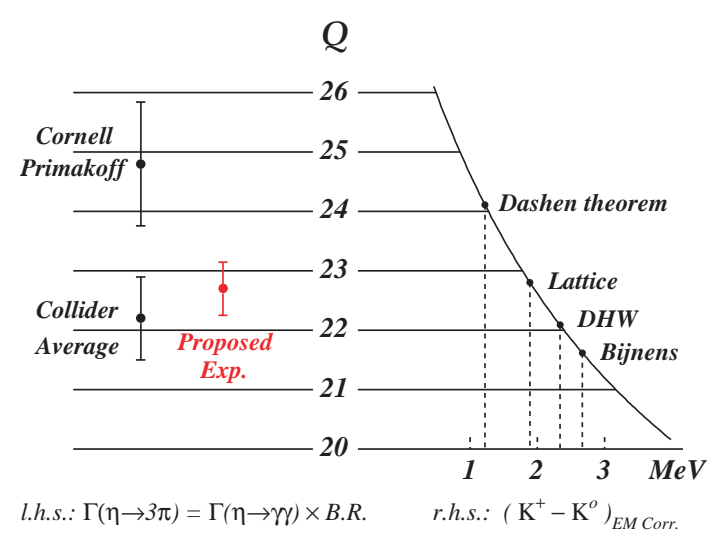

Figure 24: Quark-mass ratio $\mathcal{Q}$ as determined from $\eta$ decay and from $K^{+}-K^{0}$ mass difference. Indicated in red is the projected uncertainty from the proposed measurement of $\Gamma_{\eta \rightarrow \gamma \gamma}$. Figure adapted from H. Leutwyler [Le96].

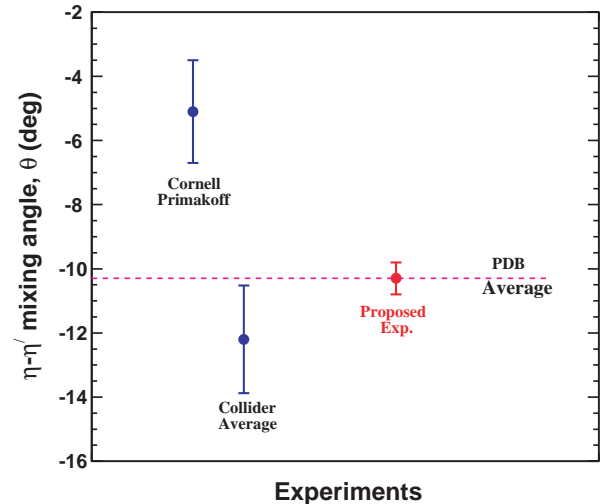

Figure 25: The $\eta-\eta^{\prime}$ mixing angle as determined by a previous Primakoff measurement, $\gamma-\gamma$ collisions in $e^{+}-e^{-}$colliders, and the projected uncertainty from the proposed measurements.

by two different methods, namely kaon mass differences and $\eta \rightarrow \pi^{+} \pi^{-} \pi^{0}$ decay width. The first method is limited by uncertainties in the theoretical calculations of the electromagnetic corrections to kaon masses. The second method has the advantage of being virtually free of electromagnetic corrections and is primarily limited by the uncertainty in the experimental determination of the $\eta \rightarrow \pi^{+} \pi^{-} \pi^{0}$ decay width, which is obtained from the $\eta \rightarrow \gamma \gamma$ decay width and the corresponding well known branching ratios. Therefore, a precise measurement of the $\eta \rightarrow \gamma \gamma$ decay width will directly impact the determination of the quark mass ratio. This decay width was obtained from previous Primakoff and from $e^{+}-e^{-}$collider experiments, which have a three- $\sigma$ discrepancy. Thus, the proposed precision Primakoff measurement of $\Gamma_{\eta \rightarrow \gamma \gamma}$ will be crucial for an accurate determination of the quark mass ratio $\mathcal{Q}$, as shown in Fig. 24.

More precise measurements of the $\eta$ and $\eta^{\prime}$ two-photon widths will impact our understanding of the role of the axial anomaly and quark masses in the $\eta^{\prime}$ meson. This information is largely contained in the $\eta-\eta^{\prime}$ mixing angle $\theta$ depicted in Fig. 25. This mixing angle depends on the $S U(3)$ breaking mass difference $m_{s}-\hat{m}$ and the mass induced on the $\eta^{\prime}$ by the axial anomaly. The latter in turn is a measure of a fundamental dynamical aspect of the QCD vacuum, namely the so called topological susceptibility, a quantity that has been recently accessible to lattice QCD calculations. At a more general level, these measurements impact the issue of whether the $\eta^{\prime}$ meson can be considered to be an approximate Goldstone Boson in the combined Chiral and $1 / N_{c}$ expansions, a question that is of great theoretical significance. It should be emphasized that determining the mixing angle from the two-photon decay widths has the advantage of being free of model dependencies that affect other determinations such as the one based on the branching ratios of the decays $\phi \rightarrow \eta \gamma$ and $\phi \rightarrow \eta^{\prime} \gamma$ [Al02]. 


\section{Upgrade Project Summary}

The key features of CEBAF that make the Upgrade so cost-effective are easily defined. By the summer of 1994, CEBAF had installed what was the world's largest superconducting radio-frequency (SRF) accelerator: an interconnected pair of antiparallel linacs, each comprising 20 cryomodules, with each cryomodule in turn containing eight SRF accelerating cavities. On average, these cavities exceed their design specifications by $50 \%$ in the two critical performance measures: accelerating gradient and $Q$. It is the success of this technology that has opened up the possibility of a relatively simple and inexpensive upgrade of CEBAF's top energy. This technological success would not be so readily multiplied if considerable foresight had not also been exercised in laying out the CEBAF tunnel "footprint", which was designed so that the magnetic arcs could accommodate an electron beam of up to $24 \mathrm{GeV}$. The latent accelerating power of the installed SRF cavities has already brought CEBAF close to $6 \mathrm{GeV}, 50 \%$ above its design energy, and recent successes in SRF development have led to the production of two cryomodules that are more than a factor of 2 more powerful than the original design. A staged development program is underway that is aimed at a cryomodule that exceeds the original specification by a factor of 5 using higher-performing sevencell cavities but fits into the same space as the original cryomodules based on five-cell cavities. The cryomodule developed as the first step in this program shows initial performance that is a factor of 4 better than the original specifications, consistent with the design goals. Using the space already available in the linac tunnels to install ten of the final-design cryomodules $12 \mathrm{GeV}$ can be attained at a modest cost.

The equipment planned for the Upgrade project takes full advantage of apparatus developed for the present program. In two of the existing halls new spectrometers are added and/or present equipment upgraded to meet the demands of the $12 \mathrm{GeV}$ program. Then a new hall, Hall D, will be added to support the meson spectroscopy program. In Hall A, the Upgrade will only add $11 \mathrm{GeV}$ capability to the beamline. This hall will be used for special setup experiments, such as the Møller experiment, and have continued use for experiments where energy resolution sufficient to separate nuclear levels is important. This remains possible with the high resolution spectrometer pair in the hall, and the capability of the upgraded accelerator to deliver beams at 2.2, 4.4, and $6.6 \mathrm{GeV}$, which are compatible with the $4 \mathrm{GeV}$ maximum momentum of these spectrometers. In Hall $\mathrm{B}$, the CEBAF Large Acceptance Spectrometer (CLAS), which was designed to study multi-particle, exclusive reactions with its combination of large acceptance and moderate momentum resolution, will be upgraded to CLAS12 and optimized for studying exclusive reactions (emphasizing the investigation of the GPD's) at high energy. It will also be used for selected valence quark structure studies involving neutron "tagging" or polarized targets capable of supporting only very low beam current. Most importantly, the maximum luminosity will be upgraded from $10^{34}$ to $10^{35} \mathrm{~cm}^{-2} \mathrm{~s}^{-1}$. The present time-of-flight counters, Čerenkov detectors, and shower counter will be retained, but the tracking system and other details of the central region of the detector will be changed to match the new physics goals. In Hall $\mathrm{C}$ a new, high-momentum spectrometer (the SHMS, Super-HighMomentum Spectrometer) will be constructed to support high-luminosity experiments detecting reaction products with momenta up to the full $11 \mathrm{GeV}$ beam energy. This feature is essential for studies such as the pion form factor, color transparency, duality, and high- $Q^{2} N^{*}$ form factors. The spectrometer will be usable at very small scattering angles. Finally, in Hall D, a tagged coherent bremsstrahlung beam and solenoidal detector will be constructed in support of a program of gluonic spectroscopy aimed at testing experimentally our current understanding that quark confinement arises from the formation of QCD flux tubes. 


\section{A The Accelerator}

The accelerator portion of the Upgrade is straightforward. The basic elements can be seen in Fig. 26, and the key parameters of the upgraded accelerator are listed in Table 1. The Upgrade utilizes the existing tunnel and does not change the basic layout of the accelerator. There are four main changes: additional acceleration in the linacs, stronger magnets for the recirculation, an upgraded cryoplant, and the addition of a tenth recirculation arc. The extra arc permits an additional "half pass" through the accelerator to reach the required $12 \mathrm{GeV}$ beam energy, followed by beam transport to Hall $\mathrm{D}$ that will be added to support the meson spectroscopy initiative.

Motivated by the science, the $12 \mathrm{GeV}$ Upgrade derives its name from the fact that it will deliver a $12 \mathrm{GeV}$ electron beam to the new end station, Hall D, where it will be used to produce $9 \mathrm{GeV}$ polarized photons for the new gluonic and $s \bar{s}$ spectroscopies. The accelerator will, in addition, be able to simultaneously send electrons of $2.2,4.4,6.6,8.8$, or $11.0 \mathrm{GeV}$ to the existing Halls $\mathrm{A}$, B, and C. The increased physics power of the present halls comes from the qualitative jump in energy and momentum transfer that the Upgrade brings, and from the enhanced instrumentation capabilities planned for the detector complements in each of them. In describing the physics in Halls A, B, and $\mathrm{C}$ we will often refer to an $11 \mathrm{GeV}$ electron beam (to be precise about the maximum beam energy available in these halls) but we will use the phrase " $12 \mathrm{GeV}$ " to describe the overall Upgrade. 


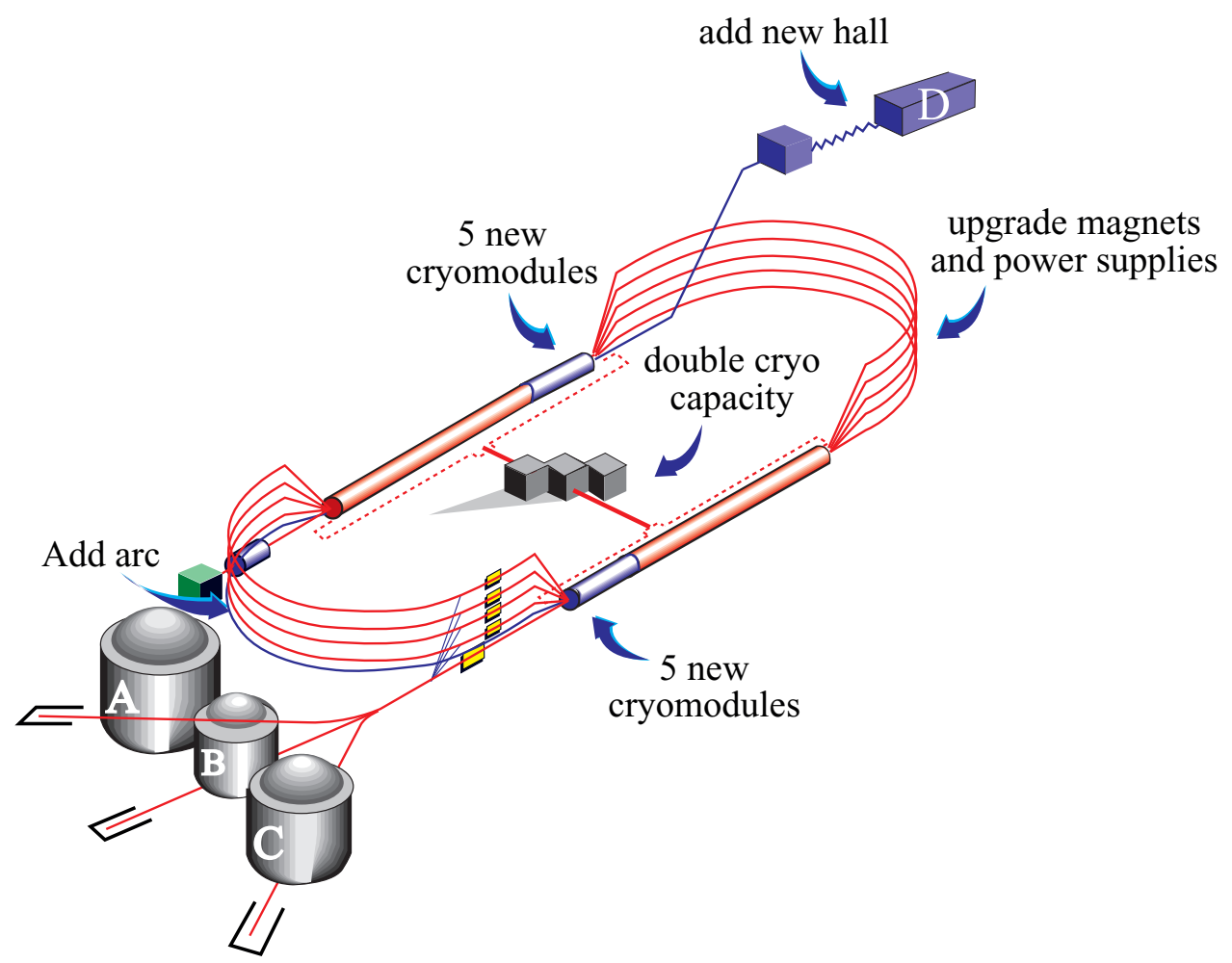

Figure 26: The configuration of the proposed $12 \mathrm{GeV}$ CEBAF Upgrade.

Table 1: Selected key parameters of the CEBAF $12 \mathrm{GeV}$ Upgrade

\begin{tabular}{ll}
\hline \hline Parameter & Specification \\
\hline Number of passes for Hall D & 5.5 (add a tenth arc) \\
Max. energy to Hall D & $12.0 \mathrm{GeV}$ (for 9 GeV photons) \\
Number of passes for Halls A, B, C & 5 \\
Max. energy to Halls A, B, C & $10.9 \mathrm{GeV}$ \\
Max. energy gain per pass & $2.2 \mathrm{GeV}$ \\
Range of energy gain per pass & $3: 1$ \\
Duty factor & $\mathrm{cw}$ \\
Max. summed current to Halls A, C* & $85 \mu \mathrm{A}$ \\
(at full, 5-pass energy) & \\
Max. summed current to Halls B, D & $5 \mu \mathrm{A}$ \\
\hline \hline
\end{tabular}

*Max. total beam power is $1 \mathrm{MW}$. 


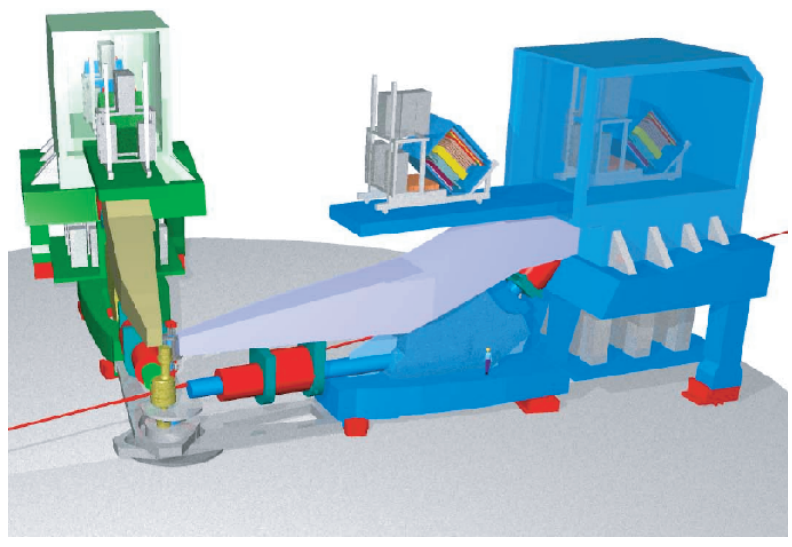

\begin{tabular}{ll}
\hline \hline Parameter & HRS \\
\hline Momentum Resolution & $1 \times 10^{-4} \mathrm{FWHM}$ \\
Momentum Range & 0.3 to $4.3 \mathrm{GeV} / \mathrm{c}$ (left) \\
& 0.3 to $3.3 \mathrm{GeV} / \mathrm{c}$ (right) \\
Momentum Acceptance & $\pm 5 \%$ \\
Horizontal Acceptance & $\pm 30 \mathrm{mrad}$ \\
Vertical Acceptance & $\pm 60 \mathrm{mrad}$ \\
Angle Range & $6^{\circ}$ to $150^{\circ}$ (left) \\
& $6^{\circ}$ to $130^{\circ}$ (right) \\
Solid Angle & $6 \mathrm{msr}$ (standard) \\
& $12 \mathrm{msr}$ (forward) \\
Vertical Resolution & $\pm 1.0 \mathrm{mrad}$ \\
Horizontal Resolution & $\pm 0.5 \mathrm{mrad}$ \\
Length Acceptance $\left(90^{\circ}\right)$ & $10 \mathrm{~cm}$ \\
Vertex Resolution & $0.1 \mathrm{~cm}$ \\
Maximum DAQ Rate & $5 \mathrm{kHz}$ \\
\hline \hline
\end{tabular}

Figure 27: A CAD drawing of the left and right Hall A High Resolution Spectrometers (HRS). Spectrometer properties are summarized in the table.

\section{B The Experimental Equipment}

Hall A and the High Resolution Spectrometers (HRS). The beamline into Hall A will be upgraded so that the hall will be able to accept the full range of beam energies avaliable after the Upgrade. Within the scope of the proposed Upgrade, Hall A will serve two major purposes. The first will be to continue the use of the high resolution spectrometer pair (see. Fig. 27) provide the lab for experiments that require high resolution, high luminosity, and the detection of reaction products with momenta between 0.4 and $4.3 \mathrm{GeV} / \mathrm{c}$. As there is already a backlog of more than five years of for such experiments, and it is expected that this line of experimental research will continue well after the Upgrade has been completed. Also, since only one of the present end stations will be able to take $11 \mathrm{GeV} / \mathrm{c}$ beam at any given time, a lower pass beam will always be available. The HRS pair is well-suited for most electromagnetic interaction experiments mounted using the 2.2, 4.4 , and $6.6 \mathrm{GeV}$ beams that provided by one-, two-, and three-pass operation. The second purpose for Hall A will be to stage major installation experiments. With a diameter of $174 \mathrm{ft}$, Hall A is the largest experimental hall at Jefferson Lab and with the ability of two HRS spectrometers to be parked at large angles the Hall can easily accommodate major installations such as the proposed parity-violation setups. 


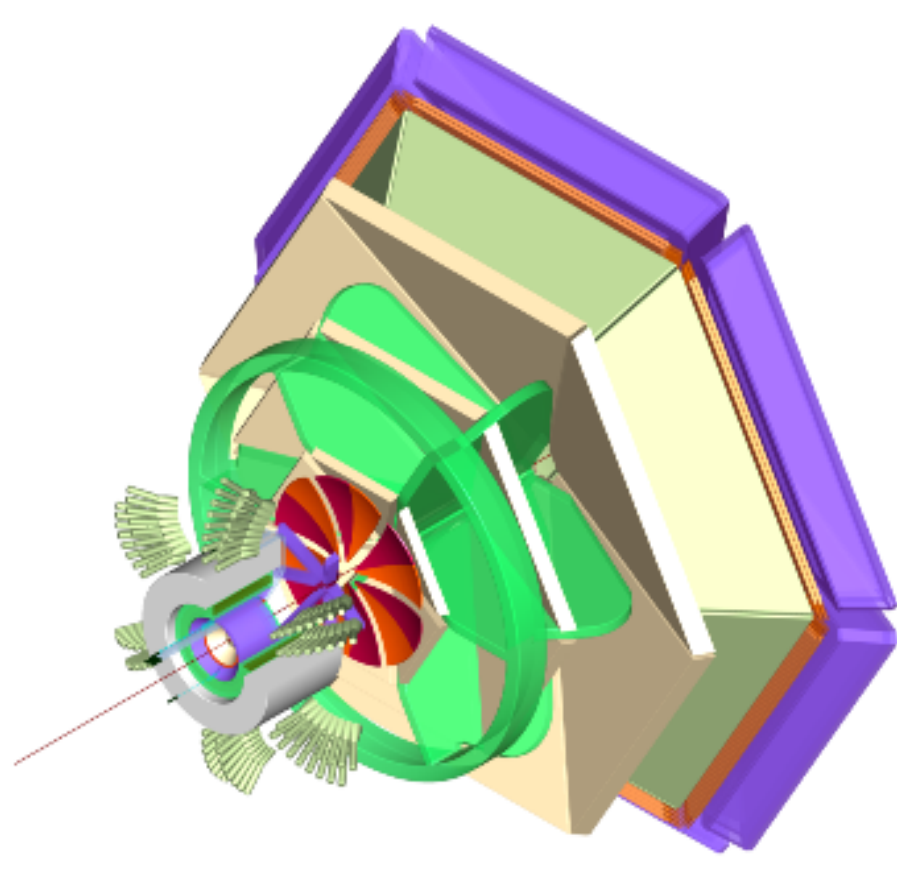

\begin{tabular}{lcr}
\hline \hline & $\begin{array}{c}\text { Forward } \\
\text { Detector }\end{array}$ & $\begin{array}{r}\text { Central } \\
\text { Detector }\end{array}$ \\
\hline Angle range & $5^{\circ}-40^{\circ}$ & $40^{\circ}-135^{\circ}$ \\
Tracks & $3^{\circ}-135^{\circ}$ & $40^{\circ}-135^{\circ}$ \\
Photons & $0.003+0.001 p$ & $\delta p_{T} / p_{T}=0.03$ \\
\hline Resolution & $<1.0$ & 8 \\
$\delta p / p$ & $<3.0$ & 2 \\
$\delta \theta(m r)$ & & \\
$\delta \phi(m r)$ & $>150$ & \\
\hline Photon detection & $4(1 \mathrm{GeV})$ & $15(1 \mathrm{GeV})$ \\
Energy (MeV) & & \\
$\delta \theta(\mathrm{mr})$ & $0.1-0.5$ & \\
\hline Neutron detection & & \\
$\eta_{e f f}$ & & \\
\hline Particle id & $100-1000$ \\
electron $/$ pion & & \\
rejection & full range & $<1.2 \mathrm{GeV} / \mathrm{c}$ \\
$\pi / P$ & full range & $<0.65 \mathrm{GeV} / \mathrm{c}$ \\
$K / \pi$ & $<4.5 \mathrm{GeV} / \mathrm{c}$ & $<0.90 \mathrm{GeV} / \mathrm{c}$ \\
$K / P$ & full range & full range \\
$\pi^{\circ} \rightarrow \gamma \gamma$ & full range & full range \\
$\eta \rightarrow \gamma \gamma$ & & \\
\hline \hline
\end{tabular}

Figure 28: The CLAS12 detector; projected acceptance and resolution are summarized in the table.

Hall B Upgrade and CLAS12. The CLAS12 detector has evolved from CLAS to meet the basic requirements for the study of the structure of nucleons and nuclei with the CEBAF $12 \mathrm{GeV}$ upgrade. A major focus of CLAS12 will be to determine the Generalized Parton Distributions (GPDs). CLAS12 will be able to carry out the core program for the study of the internal dynamics and 3D imaging of the nucleon, and quark hadronization processes. These studies are carried out by measuring exclusive, and semi-inclusive processes from hydrogen and nuclear polarized and unpolarized targets. The access to GPDs at high momentum transfer requires use of large acceptance detectors capable of operating at high luminosity. Moreover, a program to access GPDs requires use of polarized solid state targets with limited luminosity capabilities. CLAS12 will provide the large acceptance to measure these processes well.

A 3-D representation of CLAS12 is shown in Fig. 28. The included table summarizes the performance parameters. The main features are:

- High operating luminosity of $L>10^{35} \mathrm{~cm}^{-2} \mathrm{~s}^{-1}$ for hydrogen targets, a more than ten-fold increase over current CLAS operating conditions.

- Detection capabilities and particle identification for forward-going high momentum charged and neutral particles. Acceptances for electrons range from about 5 degrees to 40 degrees. Electron and pion identification is extended to cover the full momentum range.

- Capability to detect charged particles and photons at large angles up to 135 degrees.

- Improved hermeticity for the detection of charged particles and photons in regions where CLAS currently has no detection capabilities. 
CLAS12 makes use of many existing detector components. Major new components include the superconducting torus coils that cover only the forward angle range, a new gas Cerenkov counter for pion identification, additions to the electromagnetic calorimeters, and the central detector. In the following the modifications and new components are briefly described.

Forward detector: The forward detector system will use several of the existing components: the low threshold gas Cerenkov counters, all electromagnetic calorimeters, and the time-of-flight scintillators. New components include the high threshold Čerenkov counter, the torus magnet, and the forward drift chambers, which will cover an angle range from 5 to 40 degrees. The large drift chambers in CLAS will be replaced by smaller ones that will cover the 5 - 40 degree angle range with a factor 2 smaller cell sizes. Additional electromagnetic calorimetry is placed in the area of the torus coils for improved hermeticity. Lead tungstate crystals with avalanche photodiode readout have emerged as a good choice for this detector. These techniques have been in use for the existing small angle calorimeter. Two of the existing detector systems, the time-of-flight system and the electromagnetic calorimeter system, need some upgrading to allow measurement of high momentum forward going particles. A pre-shower detector will be inserted in front of the existing CLAS electromagnetic calorimeters to allow high energy photon detection. Improved particle identification will be accomplished by several means: the timing resolution of the scintillation counters will be improved by cutting the existing scintillator material into smaller slabs, and by adding an additional layer of scintillators, re-using the existing large angle panels, and by replacing the PMTs by new ones with better timing characteristics.

Central detector: A main component is a compact superconducting solenoid magnet, which has a triple function: it provides magnetic shielding of all tracking detectors from charged electromagnetic background, mostly Møller electrons and from secondary interaction. It provides the magnetic field for the momentum analysis of charged particles at large angles, and it provides the uniform 5 Tesla field needed for the operation of a dynamically polarized solid state target. The CD will detect charged particles and photons from 40 degrees to 135 degrees. A combination of tracking chambers and silicon microstrip layers will provide momentum analysis. Particle identification is achieved by time-of-flight counters. A compact calorimeter made from scintillating fibers embedded in tungsten powder will allow neutral particle detection.

Beamline and targets: Most of the existing beamline components will be re-used without changes. This includes the photon tagger and pair spectrometer, the polarized photon instrumentation, a polarized target for photon experiments, and beam position and current monitors. A beam chicane will be implemented to allow operation of transverse polarized targets for electron scattering. The chicane is expected to exist before the upgrade. 
Hall C and the Super High Momentum Spectrometer (SHMS). At a 12-GeV Jefferson Lab, Hall C will provide a new magnetic spectrometer, the Super High Momentum Spectrometer (SHMS), powerful enough to analyze charged particles with momenta approaching that of the highest energy beam. Together with its companion, the existing High Momentum Spectrometer (HMS), this will make Hall $\mathrm{C}$ the only facility in the world capable of studying (deep) exclusive reactions up to the highest momentum transfers, $Q^{2} \simeq 18(\mathrm{GeV} / \mathrm{c})^{2}$, with appropriate high luminosity. By extension, only Hall $\mathrm{C}$ will be able to fully exploit semi-exclusive reactions in the critical region where the electro-produced hadron carries almost all of the transfered energy .

Charged particles with such high momenta are boosted by relativistic kinematics into the forward detection hemisphere. Therefore, the SHMS is designed to achieve angles down to $5.5^{\circ}$, and up to $40^{\circ}$. The SHMS will cover a solid angle up to $5 \mathrm{msr}$, and boasts a large momentum and target acceptance. The existing HMS complements SHMS well, with a solid angle of up to $10 \mathrm{msr}$, an angular range between $10.5^{\circ}$ and $90^{\circ}$, and a maximum momentum of $7.3 \mathrm{GeV} / \mathrm{c}$.

Hall C's magnetic spectrometer pair will be rigidly connected to a central pivot that permits rapid, remote angle changes and reproducible rotation characteristics, which simplify accurate measurements such as Rosenbluth-type separations. From its inception, the SHMS momentum and target acceptances were designed to be large and nearly uniform, allowing for both fast and accurate data collection. The SHMS could operate at a luminosity of $10^{39} \mathrm{~cm}^{-2} \mathrm{~s}^{-1}$, with a $32 \%$ momentum acceptance.

Figure 29 shows the new SHMS spectrometer installed in the hall along with the existing HMS spectrometer. The projected performance of the SHMS for $7-\mathrm{GeV}$ running is summarized in the included table.

With the new equipment, Hall $\mathrm{C}$ will be able to deliver, amongst others:

- Nucleon elastic and transition form factors up to $Q^{2} \simeq 18(\mathrm{GeV} / \mathrm{c})^{2}$,

- Real Compton Scattering up to $s \simeq 20 \mathrm{GeV}^{2}$,

- Deep exclusive pion and kaon electroproduction up to $Q^{2} \simeq 10(\mathrm{GeV} / \mathrm{c})^{2}$, including precise longitudinal-transverse separations and spin-dependent measurements.

- A charged pion form factor measurement up to $Q^{2}=6(\mathrm{GeV} / \mathrm{c})^{2}$.

- Complete separation of the $F_{L}, F_{T}, g_{1}$, and $g_{2}$ inclusive structure functions of the proton (in the valence quark region) up to $Q^{2} \simeq 10(\mathrm{GeV} / \mathrm{c})^{2}$.

- Precision measurements of the $Q^{2}$-dependence of nuclear effects in both inclusive structure functions and (deep) exclusive scattering, crossing the charm threshold. 


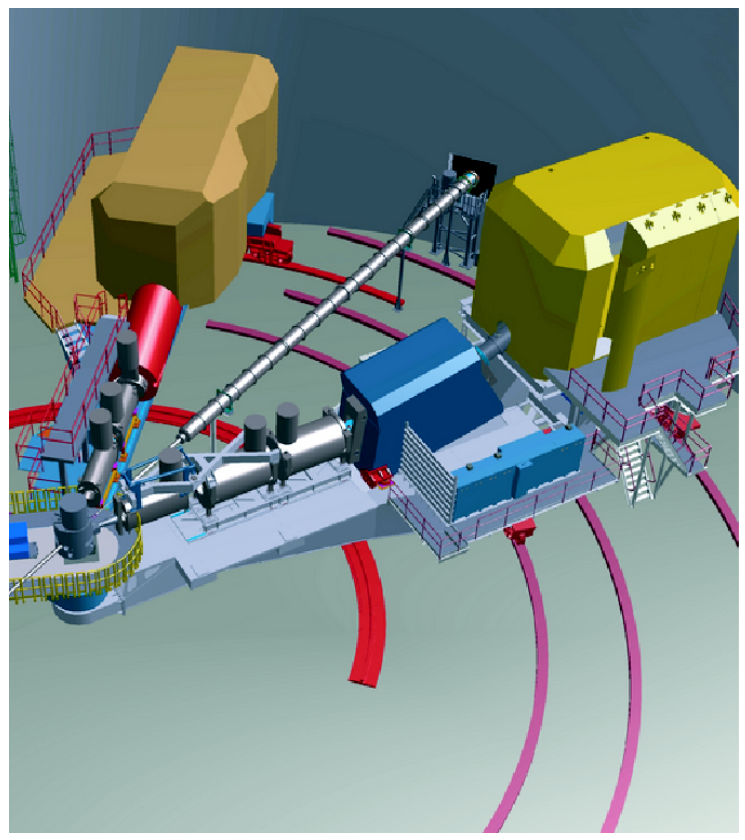

\begin{tabular}{ll}
\hline \hline Parameter & $\begin{array}{l}\text { SHMS Projections } \\
\text { (@ 7 Ge } / \text { c })\end{array}$ \\
\hline $\begin{array}{l}\text { Range of Central } \\
\text { Momentum }\end{array}$ & 2 to $11 \mathrm{GeV} / \mathrm{c}$ \\
\hline Momentum Acceptance & $-10 \%$ to $+22 \%$ \\
\hline Momentum Resolution & $0.03-0.08 \%$ \\
\hline Scattering Angle Range & 5.5 to 40 degrees \\
\hline $\begin{array}{l}\text { Vertex Length } \\
\text { (viewed at 90 degrees) }\end{array}$ & $30 \mathrm{~cm}$ \\
\hline $\begin{array}{l}\text { Horizontal Angle } \\
\text { Acceptance }\end{array}$ & $\pm 24 \mathrm{mrad}$ \\
\hline $\begin{array}{l}\text { Vertical Angle } \\
\text { Acceptance }\end{array}$ & $\pm 55 \mathrm{mrad}$ \\
\hline $\begin{array}{l}\text { Solid Angle } \\
\text { Acceptance }\end{array}$ & $5.0 \mathrm{msr}$ \\
\hline $\begin{array}{l}\text { Horizontal Angle } \\
\text { Resolution (yptar) }\end{array}$ & $0.5-1.2 \mathrm{mrad}$ \\
\hline $\begin{array}{l}\text { Vertical Angle } \\
\text { Resolution (xptar) }\end{array}$ & $0.3-1.1 \mathrm{mrad}$ \\
\hline $\begin{array}{l}\text { Vertex Length } \\
\text { Resolution (ytar) }\end{array}$ & $0.1-0.3 \mathrm{~cm}$ \\
\hline \hline
\end{tabular}

Figure 29: A CAD drawing of the new SHMS spectrometer installed in Hall C together with the existing HMS and SOS spectrometers and a summary of projected performance for the SHMS. 
Hall D and the GlueX Experiment. The GlueX experiment will be housed in a new aboveground experimental hall (Hall D) located at the east end of the CEBAF north linac. A collimated beam of linearly polarized photons (with $40 \%$ polarization) of energy 8.5 to $9 \mathrm{GeV}$ will be produced via coherent bremsstrahlung with $12 \mathrm{GeV}$ electrons. This requires thin diamond crystal radiators (no more than $20 \mu$ thick). The scattered electron from the bremsstrahlung will be tagged with sufficient precision to determine the photon energy to within $0.1 \%$.

The GlueX detector (see Fig. 30) uses an existing $2.25 \mathrm{~T}$ superconducting solenoid that is currently being refurbished. An existing 3000-element lead-glass electromagnetic calorimeter will be reconfigured to match the downstream aperture of the solenoid. The collaboration is currently looking at a DIRC design based on the SLAC BaBar detector for the Cerenkov system. Design work is being carried out with consultation with experts from SLAC. The DIRC Cherenkov Counter is followed by a scintillator time-of-flight (TOF) wall which in turn is followed by a lead glass calorimeter for detecting photons. Inside the full length of the solenoid, a lead and scintillating fiber electromagnetic calorimeter will provide position and energy measurement for photons and TOF information for charged particles. A simple start counter will surround the $30 \mathrm{~cm}$ long liquid hydrogen target. This in turn will be surrounded by a cylindrical straw-tube drift-chambers which will fill the region between the target and the cylindrical calorimeter. Planar drift chambers will be placed inside the solenoid downstream of the target to provide accurate track reconstruction for charged particles going in the forward direction.

This detector configuration has $4 \pi$ hermeticity and momentum/energy and position information for charged particles and photons produced from incoming $9 \mathrm{GeV}$ photons. This detector has been carefully optimized to carry out partial wave analysis of many particle final states. Extensive Monte Carlo studies for a wide variety of final states have been carried out to certify the design parameters and the suitability of the detector for carrying out the final analysis. In particular, the acceptance of the detector for final state particles is typically above $95 \%$ and is quite uniform over the detector. A well understood acceptance is crucial to being able to carry out precision partial wave analysis.

An active program of $R \& D$ has been underway now for several years on each of the detector subsystems. Rocking curve measurements of diamond wafers have been performed in the UK, and the coherent bremsstrahlung technique has been successfully demonstrated in Hall B at Jefferson Lab. Prototypes of tracking elements and the cylindrical calorimeter have been built and more are planned. Beam tests in Russia on TOF prototypes have resulted in a finalized design. Prototype flash ADCs have been built and tested as have TDCs based on F1 chips. Work on optimizing electronics continues and more beam tests are planned. The magnet is currently being refurbished at the Indiana University Cyclotron Facility.

The GlueX detector has undergone several reviews carried out by experts from outside the JLab community. The electronics was reviewed in the summer of 2003, while the entire detector was carefully looked at in the Fall of 2004. Finally, a review was performed on the refurbished magnet. All of these reviews have led to both improvements and optimizations of the GlueX detector. The primary characteristics of the detector are summarized in the table in Fig. 30. The hermetic design for the detector makes it an ideal tool to determine the masses and quantum numbers of mesons in the mass range of 1.5 to $2.5 \mathrm{GeV} / \mathrm{c}^{2}$. The detector will be sensitive to hybrid mesons produced with cross sections as low as a few percent of well known mesons and can also be used to map out both normal mesons and the poorly known spectra of $s \bar{s}$ mesons. 


\section{GlueX Detector}

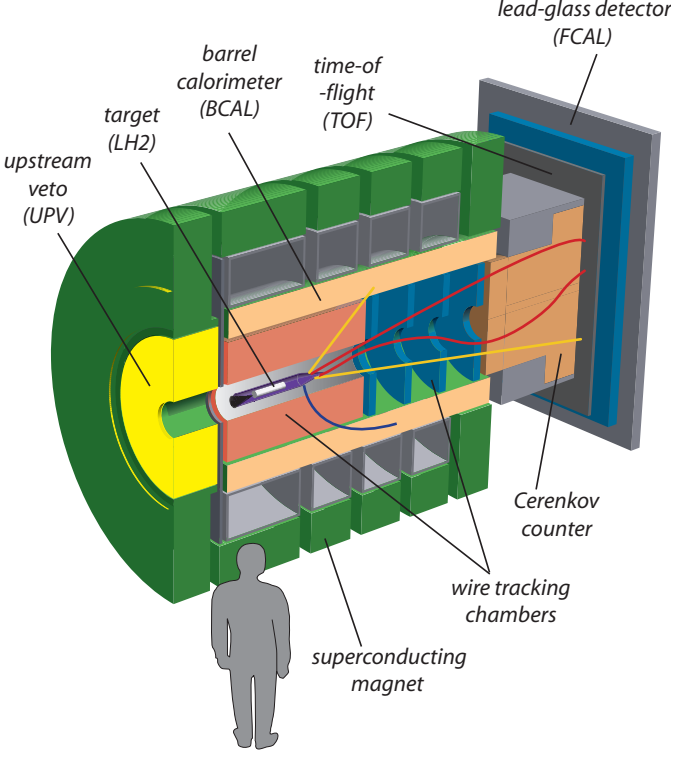

\begin{tabular}{|c|c|}
\hline Parameter & GlueX Performance \\
\hline \multicolumn{2}{|c|}{ Charged Particle Detection } \\
\hline Coverage & $1^{\circ}<\theta<170^{\circ}$ \\
\hline Momentum Resolution & $\sigma(p) / p=1-2 \%$ \\
\hline Position Resolution: & $\sigma=150-200 \mu m$ \\
\hline $\mathrm{dE} / \mathrm{dx}$ measurements: & $20<\theta<140^{\circ}$ \\
\hline BCAL time resolution: & $\sigma(t)=250 p s$ \\
\hline TOF time resolution: & $\sigma(t)=60 p s$ \\
\hline$\pi / K$ Separation (Cerenkov) & $\theta<14^{\circ}$ \\
\hline \multicolumn{2}{|c|}{$\begin{array}{ll}\text { Photon Detection } \\
\end{array}$} \\
\hline Energy measurments: & $2 \leq \theta \leq 120^{\circ}$ \\
\hline Veto: & $\theta \geq 120^{\circ}$ \\
\hline BCAL resolution $\left(E_{\gamma} \geq 20 \mathrm{MeV}\right)$ : & $\sigma(E) / E=(2+5 / \sqrt{E}) \%$ \\
\hline BCAL resolution $\left(E_{\gamma} \geq 100 \mathrm{MeV}\right)$ : & $\sigma(E) / E=(3.6+7.3 / \sqrt{E}) \%$ \\
\hline BCAL position resolution & $\sigma(x)=1 \mathrm{~cm}$ \\
\hline LGD position resolution & $\sigma(x)=1 \mathrm{~cm}$ \\
\hline \multicolumn{2}{|c|}{$\begin{array}{l}\text { DAQ/Trigger } \\
\end{array}$} \\
\hline Level 1: & $200 k H z$ \\
\hline Event Rate: & $15 \mathrm{kHz}$ to tape \\
\hline Data Rate: & $100 \mathrm{MB} / \mathrm{s}$ \\
\hline \multicolumn{2}{|c|}{ Electronics } \\
\hline Fully pipe-lined & FADCs and TDCs \\
\hline \multicolumn{2}{|c|}{ Photon Flux } \\
\hline Initially: $10^{7} \gamma / \mathrm{s}$ & Final: up to $10^{8} \mathrm{\gamma} / \mathrm{s}$ \\
\hline
\end{tabular}

Figure 30: The GlueX detector in HallD. The detector is nearly hermetic for both charged particles and photons. The figure shows a sliced open view of the detector with all elements visible. The anticipated detector performance is summarized in the table. 


\section{Appendix: PAC30 Recommendations for $12 \mathrm{GeV}$ Experiments}

Table 2: PAC 30 Recommendations (approved and conditionally approved experiments)

\begin{tabular}{|c|c|}
\hline Proposal & Title \\
\hline PR12-06-101 & Measurement of the Charged Pion Form Factor to High $Q^{2}$ \\
\hline PR12-06-102 & $\begin{array}{l}\text { Mapping the Spectrum of Light Quark Mesons and Gluonic Excitations with } \\
\text { Linearly Polarized Photons }\end{array}$ \\
\hline PR12-06-104 & $\begin{array}{l}\text { Measurement of the Ratio } \mathrm{R}=\sigma_{L} / \sigma_{T} \text { in Semi-Inclusive Deep-Inelastic Scat- } \\
\text { tering }\end{array}$ \\
\hline PR12-06-105 & $\begin{array}{l}\text { Inclusive Scattering from Nuclei at } x>1 \text { in the quasielastic and deeply } \\
\text { inelastic regimes }\end{array}$ \\
\hline PR12-06-106 & $\begin{array}{l}\text { Study of color Transparency in Exclusive Vector Meson Electroproduction } \\
\text { off Nuclei }\end{array}$ \\
\hline PR12-06-107 & The Search for Color Transparency at $12 \mathrm{GeV}$ \\
\hline PR12-06-108 & Hard Exclusive Electroproduction of $\pi^{0}$ and $\eta$ with CLAS12 \\
\hline PR12-06-109 & The Longitudinal spin Structure of the Nucleon \\
\hline PR12-06-110 & $\begin{array}{l}\text { Measurement of Neutron Spin Asymmetry } A_{1}^{n} \text { in the Valence Quark Region } \\
\text { Using an } 11 \mathrm{GeV} \text { Beam and a Polarized }{ }^{3} \mathrm{He} \text { Target in Hall } \mathrm{C}\end{array}$ \\
\hline PR12-06-112 & $\begin{array}{l}\text { Probing the Protons Quark Dynamics in Semi-Inclusive Pion Production at } \\
12 \mathrm{GeV}\end{array}$ \\
\hline PR12-06-113 & The Structure of the Free Neutron at Large $x_{B j o r k e n}$ \\
\hline PR12-06-114 & $\begin{array}{l}\text { Measurement of Electron-Helicity Dependent Cross Sections of Deeply Vir- } \\
\text { tual Compton Scattering with CEBAF at } 12 \mathrm{GeV}\end{array}$ \\
\hline PR12-06-117 & Quark Propagation and Hadron Formation \\
\hline PR12-06-118 & $\begin{array}{l}\text { Measurement of the } F_{2}^{n} / F_{2}^{p}, d / u \text { Ratios and } A=3 \text { EMC Effect in Deep } \\
\text { Inelastic Scattering off the Tritium and Helium Mirror Nuclei }\end{array}$ \\
\hline PR12-06-119 & Deeply Virtual Compton Scattering with CLAS12 at $11 \mathrm{GeV}$ \\
\hline PR12-06-121 & $\begin{array}{l}\text { A Path to Color Polarizabilities in the Neutron: A Precision Measurement } \\
\text { of the Neutron } g_{2} \text { and } d_{2} \text { at High } Q^{2} \text { in Hall C }\end{array}$ \\
\hline PR12-06-122 & $\begin{array}{l}\text { Measurement of neutron asymmetry } A_{l}^{n} \text { in the valence quark region using } \\
8.8 \mathrm{GeV} \text { and } 6.6 \mathrm{GeV} \text { beam energies and Bigbite spectrometer in Hall A }\end{array}$ \\
\hline
\end{tabular}




\section{References}

[Ac98] K. Ackerstaff et al. [HERMES Collaboration], Phys. Rev. Lett. 81, 5519 (1998).

[Ad01] C. Adloff, et. al. [H1 collaboration], Phys. Lett. B 517, 47 (2001)

[AF00] I. R. Afnan et al., Phys. Lett. B 493, 36 (2000).

[Af03] I. R. Afnan et al., Phys. Rev. C 68, 035201 (2003).

[Ai01] A. Airapetian et al., [HERMES collaboration], Phys. Rev. Lett. 87, 182001 (2001)

[Al02] A. Aloisio et al., Phys. Lett. B 541, 45 (2002)

[Am91] P. Amaudraz et al., Phys. Rev. Lett. 66, 2712 (1991).

[Am04] M. Amarian et al. [Jefferson Lab E94-010 Collaboration], Phys. Rev. Lett. 92, 022301 (2004).

[An95] M. Anselmino, A. Efremov and E. Leader, Phys. Rept. 261, 1 (1995). [Erratum-ibid. 281, 399 (1997)].

[An76] R. L. Anderson et al., Phys. Rev. D14, 679 (1976); G. White et al., Phys. Rev. D49, $58(1994)$

[Ba94] A. Baldit et al., Phys. Lett. B 332, 244 (1994).

[Ba01] V. Barone, A. Drago and P. G. Ratcliffe, Phys. Rept. 359, 1 (2002).

[Ba02] S. D. Bass and A. De Roeck, Nucl. Phys. Proc. Suppl. 105, 1 (2002).

[Be03] A. Belitsky, X. Ji, and F. Yuan, Phys.Rev.Lett. 91, 092003 (2003).

[Be03a] A. V. Belitsky and D. Muller, Phys. Rev. Lett. 90, 022001 (2003).

[Be05] A. V. Belitsky and A. Radyushkin, "Unraveling hadron structure with generalized parton distributions," (submitted to Phys. Rep.) (available as JLAB-THY-04-34 at https:// www1.jlab.org/Ul/Publications/view_pub.cfm?pub_id=5595.

[Br79] S. J. Brodsky and G. P. Lepage, Phys. Lett. B87, 359 (1979).

[Br80] S. J. Brodsky and G. P. Lepage, Phys. Rev. D 22, 2157 (1980).

[Br01] S. J. Brodsky, E. Chudakov, P. Hoyer and J.-M. Laget, Phys. Lett. B498, 23 (2001).

[Br02] S. Brodsky et al., Phys.Lett. B 530, 99 (2002).

[Bu02] M. Burkardt, Int. J. Mod. Phys. A 18, 173 (2003).

[Bu02a] M. Burkardt, Phys. Rev. D 66, 114005 (2002).

[Bu03] M. Burkardt, Int. J. Mod. Phys. A 18, 173 (2003).

[Cl88] F. E. Close and A. W. Thomas, Phys. Lett. B 212, 227 (1988). 
[C195] F. E. Close, private communication, advocates using the positive term "strong QCD" instead of the negative "nonperturbative QCD".

[Co02] J. Collins, hep-ph/0204004 (2002)

[Co93] J. Collins, Nucl.Phys.B396, 161 (1993)

[Co97] J. C. Collins, L. Frankfurt, and M. Strikman, Phys. Rev. D 56, 2982 (1997).

[De02] W. Detmold, W. Melnitchouk and A. W. Thomas, Phys. Rev. D 66, 054501 (2002), and references therein.

[Di96] D. Diakonov, V. Petrov, P. Pobylitsa, M. V. Polyakov and C. Weiss, Nucl. Phys. B 480, 341 (1996).

[Di99] M.Diehl, T.Feldmann, R.Jakob and P.Kroll, Eur. Phys. J. C 8409 (1999). M.Diehl, T.Feldmann, R.Jakob and P.Kroll, Phys. Lett. B 460 , 204, (1999).

[Di02] M. Diehl, Eur. Phys. C 25, 223 (2002)

[Di03] M. Diehl, Phys. Rept. 388, 41 (2003).

[Do02] D. Dolgov et al. [Lattice Hadron Physics Collaboration], Phys. Rev. D 66, 034506 (2002).

[Dr05] J. Dreschler, et al. [HERMES Collaboration], AIP Conf. Proc. 842, 375 (2005).

[Ef80] A. V. Efremov and A. V. Radyushkin, Theor. Math. Phys. 42, 97 (1980).

[Ef04] A. V. Efremov, K. Goeke, S. Menzel, A. Metz and P. Schweitzer, arXiv:hep-ph/0412353.

[Fi77] R. D. Field and R. P. Feynman, Phys. Rev. D 15, 2590 (1977).

[Fr01] A. Freund, M. McDermott, and M. Strikman, arXiv:hep-ph/0208160

[Fr02] L. Frankfurt and M. Strikman, Phys. Rev. D 66, 031502 (2002).

[Fr88] L. L. Frankfurt and M. I. Strikman, Phys. Rep. 160, 235 (1988).

[Fr99] L. L. Frankfurt, V. Polyakov, M. Strikman and M. Vanderhaeghen, Phys. Rev. Lett. 84, 2589 (2000).

[Fr99a] L. L. Frankfurt, M. V. Polyakov, M. Strikman and M. Vanderhaeghen, Phys. Rev. Lett. 84, 2589 (2000).

[Fr04] L. Frankfurt, M. Strikman and C. Weiss, Phys. Rev. D 69, 114010 (2004); L. Frankfurt et al., hep-ph/0412260.

[Ga01] G. T. Garvey and J.-C. Peng, Prog. Part. Nucl. Phys. 47, 203 (2001).

[Ga01b] H. Gao, T.S.-H. Lee and V. Marinov, Phys. Rev. C 63, R022201 (2001).

[Go01] K. Goeke, V. Polyakov and M. Vanderhaeghen, Prog. Part. Nucl. Phys. 47, 401 (2001).

[Gr01] V. Yu. Grishina et al., Euro. Journal of Phys. A 10, 355 (2001).

[Gu03] M. Guidal and M. Vanderhaeghen, Phys. Rev. Lett. 90, 012001 (2003). 
[Gu04] M. Guidal, M. V. Polyakov, A. V. Radyushkin and M. Vanderhaeghen, arXiv:hep$\mathrm{ph} / 0410251$.

[Gu04a] P. A. M. Guichon and A. W. Thomas, Phys. Rev. Lett. 93, 132502 (2004),

[Ha98] E. A. Hawker et al., Phys. Rev. Lett. 80, 3715 (1998); R. S. Towell et al., Phys. Rev. D 64, 052002 (2001).

[Ha04] D. J. Hamilton et al. [Jefferson Lab Hall A Collaboration], arXiv:nucl-ex/0410001.

[Hu00] H. W. Huang and P. Kroll, Eur. Phys. J. C17, 433 (2000); P. Kroll, hep-ph/0207118 (2002).

[Hu02] H. W. Huang, P. Kroll and T. Morii, Eur. Phys. J. C 23, 301 (2002) [Erratum-ibid. C 31, 279 (2003)].

[Hu03] H.W. Huang et al., hep-ph/0309071.

[Id04] A. Idilbi, X. Ji and J. P. Ma, Phys. Rev. D 69, 014006 (2004).

[Is91] N. Isgur and M. B. Wise, Phys. Rev. D 43, 819 (1991).

[Is99] N. Isgur, Phys. Rev. D 59, 034013 (1999).

[Ji97] X. Ji, Phys. Rev. Lett. 78, 610 (1997); Phys. Rev. D 55, 7114 (1997).

[Ji03] X. Ji, Phys. Rev. Lett. 91, 062001 (2003).

[Ka99] S. S. Kamalov and S. N. Yang, Phys. Rev. Lett. 83, 4494 (1999).

[Ko93] L. A. Kondratyuk et al., Phys. Rev. C 482491 (1993).

[Ku02] A. Kurylov, M. J. Ramsey-Musolf, and S. Su, "Parity-violating electron scattering as a probe of supersymmetry", hep-ph/0205183 (2002).

[Le96] H. Leutwyler, Phys. Lett. B378 313 (1996).

[Me96] W. Melnitchouk and A. W. Thomas, Phys. Lett. B377, 11 (1996).

[Me97] W. Melnitchouk, M. Sargsian, and M. I. Strikman, Z. Phys. A359, 359 (1997).

[Me99] W. Melnitchouk, J. Speth and A.W. Thomas, Phys. Rev. D 59, 014033 (1999).

[Me05] W. Melnitchouk, R. Ent and C. Keppel, arXiv:hep-ph/0501217.

[Mu96] P.J. Mulders and R.D. Tangerman, Nucl.Phys.B461, 197(1996)

[Na88] J. Napolitano et al., Phys. Rev. Lett. 61, 2530 (1988); S.J. Freedman et al., Phys. Rev. C 48, 1864 (1993); J.E. Belz et al., Phys. Rev. Lett. 74, 646 (1995); C. W. Bochna et al., Phys. Rev. Lett. 81, 4576 (1988).

[NA99] Nuclear Physics: The Core of Matter, The Fuel of Stars, The Committee on Nuclear Physics of the Board on Physics and Astronomy of the Commission on Physical Sciences, Mathematics, and Applications of the National Research Council, National Academy Press, Washington, D.C. (1999). 
[NS02] "Opportunities in Nuclear Science: A Long-Range Plan for the Next Decade", a report by the DOE/NSF Nuclear Science Advisory Committee, April, 2002.

[Pa01] E. Pace, G. Salme and S. Scopetta, Nucl. Phys. A 689, 453 (2001); E. Pace, G. Salme, S. Scopetta and A. Kievsky, Phys. Rev. C 64, 055203 (2001).

[pCDR] Pre-Conceptual Design Report (pCDR) for the Science and Experimental Equipment for the $12 \mathrm{GeV}$ upgrade of $C E B A F$, an internal report of the Thomas Jefferson National Accelerator Facility, Newport News, VA (2004) (available at http://www.jlab.org/ div_dept/physics_division/pCDR_public/pCDR_final/).

[Po03] M. V. Polyakov, Phys. Lett. B 555, 57 (2003).

[Po03a] P. V. Pobylitsa, arXiv:hep-ph/0301236.

[Pr78] C. Y. Prescott, et al., Phys. Lett. B77, 347 (1978).

[Ra97] A. Radyushkin, Phys.Lett.B380.417 (1996); Phys. Rev. D 56, 5524 (1997).

[Ra98] A. V. Radyushkin, Phys. Rev. D 58, 114008 (1998).

[Rapc] Radyushkin A (private communication).

[Sa01] M. M. Sargsian, S. Simula and M. I. Strikman, Phys. Rev. C 66, 024001 (2002).

[Sa03] P.R. Saull [ZEUS collaboration] arXiv:hep-ex/0003030

[Sc91] A. W. Schreiber, A. I. Signal and A. W. Thomas, Phys. Rev. D 44, 2653 (1991).

[Sc01] E. C. Schulte, et al., Phys. Rev. Lett. 87, 102302 (2001).

[St01] S. Stepanyan, et al. [CLAS collaboration], Phys. Rev. Lett. 87, 182002-1 (2001)

[St03a] M. Strikman and C. Weiss, Phys. Rev. D 69, 054012 (2004).

[St03b] P. Stoler, Phys. Rev. Lett. 91, 172303 (2003).

[St03d] P. Stoler, Phys. Rev. Lett. 91, 172303 (2003).

[Th83] A. W. Thomas, Phys. Lett. B 126, 97 (1983).

[Th01] A. W. Thomas and W. Weise, "The Structure of the Nucleon," Berlin, Germany: WileyVCH (2001).

[Va99 M. Vanderhaeghen, P. A. M. Guichon, and M. Guidal, Phys. Rev. D 60, 094017 (1999).

[Wh92] L. Whitlow et al., Phys. Lett. B282, 475 (1992).

[Wi99] R. Windmolders, Nucl. Phys. Proc. Suppl. 79, 51 (1999).

[WP01] "The Science Driving the $12 \mathrm{GeV}$ Upgrade of CEBAF", an internal report of the Thomas Jefferson National Accelerator Facility, Newport News, VA (2001)

[Zh03] L.Y. Zhu et al., Phys. Rev. Lett. 91, 022003 (2003).

[Zh03a] X. Zheng et al. [Jefferson Lab Hall A Collaboration], Phys. Rev. Lett. 92, 012004 (2004).

[Zh04] X. Zheng et al. [Jefferson Lab Hall A Collaboration], Phys. Rev. C 70, 065207 (2004). 